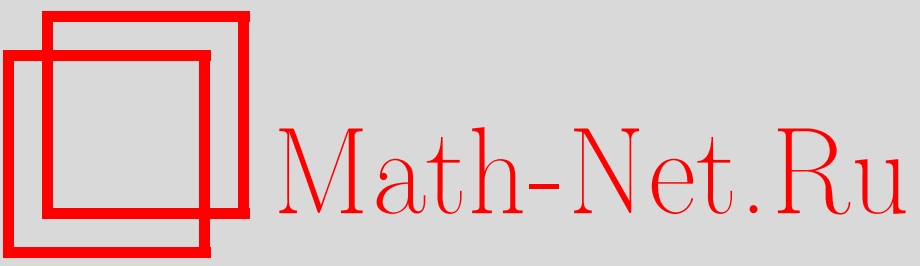

А. Н. Сергеев, Оператор Калоджеро и супералгебры Ли, ТМФ, 2002, том 131, номер 3, 355-376

DOI: https://doi.org/10.4213/tmf334

Использование Общероссийского математического портала Math-Net.Ru подразумевает, что вы прочитали и согласны с пользовательским соглашением

http://www.mathnet.ru/rus/agreement

Параметры загрузки:

IP : 3.89.197.203

26 апреля 2023 г., 16:49:27 
ТЕОРЕТИЧЕСКАЯ

И МАТЕМАТИЧЕСКАЯ

ФИЗИКА

Том 131, № 3

июнь, 2002

(C) 2002 г.

А.Н. Сергеев*

\title{
ОПЕРАТОР КАЛОДЖЕРО И СУПЕРАЛГЕБРЫ ЛИ
}

\begin{abstract}
Построен супераналог оператора Калоджеро $\mathcal{S} \mathcal{L}$, зависящий от параметра $k$. Он связан с системой корней супералгебры Ли $\mathfrak{g l}(n \mid m)$. Для $m=0$ это обычный оператор Калоджеро, а для $m=1$, с точностью до замены переменных и параметра $k$, это оператор, построенный Веселовым, Чалых и Фейгиным. Для $k=1,1 / 2$ оператор $\mathcal{S} \mathcal{L}$ является радиальной частью оператора Лапласа второго порядка для симметрических суперпространств, соответствующих парам $(\mathfrak{g l} \oplus \mathfrak{g l}, \mathfrak{g l}),(\mathfrak{g l}, \mathfrak{o s p})$. Показано, что при любых $m$ и $n$ супераналоги полиномов Джека, построенные Керовым, Окуньковым и Ольшанским, являются собственными функциями оператора $\mathcal{S} \mathcal{L}$. Для $k=1,1 / 2$ супераналоги полиномов Джека совпадают со сферическими функциями на суперпространствах. Изучается также алгебраический аналог интеграла Березина.
\end{abstract}

\section{1. ВВЕДЕНИЕ}

Данная работа является подробным изложением статьи [1]. В настояшей работе определен и исследован супераналог оператора Калоджеро, связанньй с системой корней супералгебры Ли $\mathfrak{g l}(n \mid m)$. Следует отметить, что построенный супераналог оператора Калоджеро зависит только от четных переменных. В работе [2] был определен супераналог оператора Калоджеро, содержаший одинаковое количество четных и нечетных переменных. В работах [3], [4] были определены и исследованы зависяшие от четных и нечетных переменных супераналоги полиномов Джека, связанные с супераналогом оператора Калоджеро, построенного в [2]. В работе [5] доказано сушествование инвариантного интеграла для классических супералгебр Ли. В разделе 7 передоказан результат работы [5] без привлечения теории супералгебр Хопфа, а также доказана двусторонняя инвариантность построенного интеграла. По-видимому, с помошью результатов работы [6] можно построить супераналоги оператора Калоджеро для других простых супералгебр Ли.

*Балаковский университет техники, технологии и контроля, Балаково, Саратовская обл., Россия. E-mail: sergeev@bittu.org.ru 
Гамильтониан квантовой задачи Калоджеро имеет вид

$$
\mathcal{L}=\sum_{i=1}^{n}\left(\frac{\partial}{\partial t_{i}}\right)^{2}-\frac{1}{2} k(k-1) \sum_{i<j} \frac{\omega^{2}}{\operatorname{sh}^{2} \frac{\omega}{2}\left(t_{i}-t_{j}\right)} .
$$

Этот оператор является частным случаем (соответствующим системе корней $R$ алгебры Ли $\mathfrak{g l}(n))$ оператора, построенного Ольшанецким и Переломовым [7]:

$$
\mathcal{L}=\Delta-\sum_{\alpha \in R^{+}} k_{\alpha}\left(k_{\alpha}-1\right) \frac{(\alpha, \alpha)}{\left(e^{\frac{\alpha}{2}}-e^{-\frac{\alpha}{2}}\right)^{2}} .
$$

Веселов, Фейгин и Чалых предложили [8], [9] следуюшее обобшение оператора (1.1):

$$
\begin{aligned}
\mathcal{L}^{\prime}= & \sum_{i=1}^{n}\left(\frac{\partial}{\partial t_{i}}\right)^{2}+\left(\frac{\partial}{\partial t_{n+1}}\right)^{2}-\frac{1}{2} k(k+1) \sum_{i<j} \frac{\omega^{2}}{\operatorname{sh}^{2} \frac{\omega}{2}\left(t_{i}-t_{j}\right)}- \\
& -\frac{1}{2}(k+1) \sum_{i=1}^{n} \frac{\omega^{2}}{\operatorname{sh}^{2} \frac{\omega}{2}\left(t_{i}-\sqrt{k} t_{n+1}\right)} .
\end{aligned}
$$

Известно [10], что собственные функции оператора (1.1) могут быть выражены через полиномы Джека $P_{\lambda}\left(x_{1}, \ldots, x_{n} ; k\right)$, где $\lambda$ - разбиение (определения и свойства полиномов Джека см. в [11], [12]). Известно также [11], что для $k=1,1 / 2,2$ (мы используем параметр $k=1 / \alpha$, обратный параметру полиномов Джека $\alpha$, который использует Макдональд [11]) полиномы Джека интерпретируются как сферические функции на симметрических пространствах, соответствуюшие парам $(\mathfrak{g l} \oplus \mathfrak{g l}, \mathfrak{g l}),(\mathfrak{g l}, \mathfrak{s p})$ и $(\mathfrak{g l} l \mathfrak{o})$. В этих случаях оператор Калоджеро является радиальной частью оператора Лапласа второго порядка.

Корни супералгебры $\mathfrak{g l}(n \mid m)$. Пусть $I=I_{\overline{0}} \amalg I_{\overline{1}}$ - объединение четных индексов $I_{\overline{0}}=\{1, \ldots, n\}$ и нечетных индексов $I_{\overline{1}}=\{\overline{1}, \ldots, \bar{m}\}$. Пусть $\operatorname{dim} V=(n \mid m)$ и $e_{1}, \ldots, e_{n}$, $e_{\overline{1}}, \ldots, e_{\bar{m}}$ - базис $V$ такой, что четность каждого вектора равна четности его индекcа. Пусть $\left\{e_{i j} \mid i, j \in I\right\}$ - базис $\mathfrak{g l}(V)$, состоящий из матричных единиц. Пусть также $\mathfrak{h}=\operatorname{Span}\left(e_{i i} \mid i \in I\right)$ - подалгебра Картана, состояшая из диагональных матриц, и $\varepsilon_{1}, \ldots, \varepsilon_{n}, \varepsilon_{\overline{1}}, \ldots, \varepsilon_{\bar{m}}-$ левый дуальный базис к $\left\{e_{i i} \mid i \in I\right\}$ в $\mathfrak{h}^{*}$. Тогда множество корней описывается следующим образом: $R=R_{11} \amalg R_{22} \amalg R_{12} \amalg R_{21}$, где

$$
\begin{array}{ll}
R_{11}=\left\{\varepsilon_{i}-\varepsilon_{j} \mid i, j \in I_{\overline{0}}\right\}, & R_{22}=\left\{\varepsilon_{i}-\varepsilon_{j} \mid i, j \in I_{\overline{1}}\right\}, \\
R_{12}=\left\{\varepsilon_{i}-\varepsilon_{j} \mid i \in I_{\overline{0}}, j \in I_{\overline{1}}\right\}, & R_{21}=\left\{\varepsilon_{i}-\varepsilon_{j} \mid i \in I_{\overline{1}}, j \in I_{\overline{0}}\right\} .
\end{array}
$$

На $\mathfrak{h}^{*}$ определим зависяшее от параметра $k$ скалярное произведение, полагая

$$
\left(v_{1}^{*}, v_{2}^{*}\right)_{k}=\sum_{i=1}^{n} v_{1}^{*}\left(e_{i i}\right) v_{2}^{*}\left(e_{i i}\right)-k \sum_{\bar{j}=1}^{m} v_{1}^{*}\left(e_{\bar{j} \bar{j}}\right) v_{2}^{*}\left(e_{\bar{j} \bar{j}}\right),
$$


и положим $\rho_{(k)}=k \rho_{1}+\rho_{2} / k-\rho_{12}$, где

$$
\rho_{1}=\frac{1}{2} \sum_{\alpha \in R_{11}^{+}} \alpha, \quad \rho_{2}=\frac{1}{2} \sum_{\beta \in R_{22}^{+}} \beta, \quad \rho_{12}=\frac{1}{2} \sum_{\gamma \in R_{12}} \gamma .
$$

Для любого $l \in \mathfrak{h}^{*}$ определим $e^{l}$ как линейный функционал на $S(\mathfrak{h})$, который продолжает $l$ до гомоморфизма $S(\mathfrak{h})$ в поле комплексных чисел, и обозначим через $\mathfrak{H}$ подалгебру в алгебре частных $S\left(\mathfrak{h}^{*}\right)$, порожденную элементами $e^{l}$ для $l \in \mathfrak{h}^{*}$ и $\left(1-e^{\alpha}\right)^{-1}$ для $\alpha \in R$. На $\mathfrak{H}$ определим операторы $\partial_{i}, \partial_{\bar{j}}$, полагая

$$
\partial_{i}\left(e^{v^{*}}\right)=v^{*}\left(e_{i i}\right) e^{v^{*}}, \quad \partial_{\bar{j}}\left(e^{v^{*}}\right)=v^{*}\left(e_{\bar{j} \bar{j}}\right) e^{v^{*}} .
$$

Определим супераналог оператора Калоджеро

$$
\begin{aligned}
\mathcal{S L}= & \sum_{i=1}^{n} \partial_{i}^{2}-k \sum_{j=1}^{m} \partial_{\bar{j}}^{2}-k(k-1) \sum_{\alpha \in R_{11}^{+}} \frac{(\alpha, \alpha)_{k}}{\left(e^{\frac{\alpha}{2}}-e^{-\frac{\alpha}{2}}\right)^{2}}+ \\
& +\frac{1}{k}\left(\frac{1}{k}-1\right) \sum_{\beta \in R_{22}^{+}} \frac{(\beta, \beta)_{k}}{\left(e^{\frac{\beta}{2}}-e^{-\frac{\beta}{2}}\right)^{2}}-2 \sum_{\gamma \in R_{12}} \frac{(\gamma, \gamma)_{k}}{\left(e^{\frac{\gamma}{2}}-e^{-\frac{\gamma}{2}}\right)^{2}}
\end{aligned}
$$

Легко проверить, что супераналог оператора Калоджеро может быть переписан в виде

$$
\begin{aligned}
\mathcal{S} \mathcal{L}= & \sum_{i=1}^{n} \partial_{i}^{2}-k \sum_{j=1}^{m} \partial_{\bar{j}}^{2}-k(k-1) \sum_{\alpha \in R_{11}^{+}} \frac{1}{\left(e^{\frac{\alpha}{2}}-e^{-\frac{\alpha}{2}}\right)^{2}}+ \\
& +\frac{2(1-k)}{k} \sum_{\beta \in R_{22}^{+}} \frac{1}{\left(e^{\frac{\beta}{2}}-e^{-\frac{\beta}{2}}\right)^{2}}-2(1-k) \sum_{\gamma \in R_{12}} \frac{1}{\left(e^{\frac{\gamma}{2}}-e^{-\frac{\gamma}{2}}\right)^{2}} .
\end{aligned}
$$

Заметим, что замена переменных $k \rightarrow-s, \varepsilon_{j} \rightarrow \sqrt{s} \varepsilon_{j}$ для $j \in I_{\overline{1}}$ переводит $\mathcal{S} \mathcal{L}$ в оператор

$$
\begin{aligned}
\overline{\mathcal{S}}= & \sum_{i=1}^{n} \partial_{i}^{2}+\sum_{\bar{j}=1}^{m} \partial_{\bar{j}}^{2}-s(s+1) \sum_{\alpha \in R_{11}^{+}} \frac{1}{\left(e^{\frac{\alpha}{2}}-e^{-\frac{\alpha}{2}}\right)^{2}}- \\
& -\frac{2(s+1)}{s} \sum_{\beta \in R_{22}^{+}} \frac{1}{\left(e^{\frac{\sqrt{s} \beta}{2}}-e^{-\frac{\sqrt{s} \beta}{2}}\right)^{2}}-2(s+1) \sum_{\gamma \in R_{12}} \frac{1}{\left(e^{\frac{\gamma_{s}}{2}}-e^{-\frac{\gamma_{s}}{2}}\right)^{2}},
\end{aligned}
$$

где $\gamma_{s}=\varepsilon_{i}-\sqrt{s} \varepsilon_{j}$, если $\gamma=\varepsilon_{i}-\varepsilon_{j}$. Отсюда следует, что если $\operatorname{dim} V_{\overline{1}}=1$, то оператор (1.8) совпадает с оператором (1.3), введенным в работе [8]. Для того чтобы описать собственные функции $\mathcal{S} \mathcal{L}$, удобно ввести оператор

$$
\begin{gathered}
\mathcal{M}=\left(\delta^{(k)}\right)^{-1}\left(L-\left(\rho_{(k)}, \rho_{(k)}\right) k\right) \delta^{(k)} \\
\delta^{(k)}=\prod_{\alpha \in R_{11}^{+}}\left(e^{\frac{\alpha}{2}}-e^{-\frac{\alpha}{2}}\right)^{k} \prod_{\beta \in R_{22}^{+}}\left(e^{\frac{\beta}{2}}-e^{-\frac{\beta}{2}}\right)^{1 / k} \prod_{\gamma \in R_{12}}\left(e^{\frac{\gamma}{2}}-e^{-\frac{\gamma}{2}}\right)^{-1} .
\end{gathered}
$$


Лемма 1.1. Следующее выражение дает явную форму оператора $\mathcal{M}$ :

$$
\mathcal{M}=\sum_{i=1}^{n} \partial_{i}^{2}-k \sum_{\bar{j}=1}^{m} \partial_{\bar{j}}^{2}+k \sum_{\alpha \in R_{11}^{+}} \frac{e^{\alpha}+1}{e^{\alpha}-1} \partial_{\alpha}-\sum_{\beta \in R_{22}^{+}} \frac{e^{\beta}+1}{e^{\beta}-1} \partial_{\beta}-\sum_{\gamma \in R_{12}} \frac{e^{\gamma}+1}{e^{\gamma}-1} \partial_{\gamma, k}
$$

әде

$$
\begin{aligned}
& \partial_{\alpha}=\partial_{i}-\partial_{j} \quad \partial \Omega \Omega \quad \alpha=\varepsilon_{i}-\varepsilon_{j}, \\
& \partial_{\beta}=\partial_{\bar{i}}-\partial_{\bar{j}} \quad \partial \Omega_{\text {S }} \quad \beta=\varepsilon_{\bar{i}}-\varepsilon_{\bar{j}}, \\
& \partial_{\gamma, k}=\partial_{i}+k \partial_{\bar{j}} \quad \partial \Omega я \quad \gamma=\varepsilon_{i}-\varepsilon_{\bar{j}} .
\end{aligned}
$$

В новых переменных $x_{i}=e^{\varepsilon_{i}}$ и $y_{j}=e^{\varepsilon_{\bar{j}}}$ оператор $\mathcal{M}$ имеет вид

$$
\begin{aligned}
\mathcal{M}= & \sum_{i=1}^{n}\left(x_{i} \frac{\partial}{\partial x_{i}}\right)^{2}-k \sum_{j=1}^{m}\left(y_{j} \frac{\partial}{\partial y_{j}}\right)^{2}+k \sum_{1 \leqslant i<j \leqslant n} \frac{x_{i}+x_{j}}{x_{i}-x_{j}}\left(x_{i} \frac{\partial}{\partial x_{i}}-x_{j} \frac{\partial}{\partial x_{j}}\right)- \\
& -\sum_{1 \leqslant i<j \leqslant n} \frac{y_{i}+y_{j}}{y_{i}-y_{j}}\left(y_{i} \frac{\partial}{\partial y_{i}}-y_{j} \frac{\partial}{\partial y_{j}}\right)- \\
& -\sum_{1 \leqslant i \leqslant n, 1 \leqslant j \leqslant m} \frac{x_{i}+y_{j}}{x_{i}-y_{j}}\left(x_{i} \frac{\partial}{\partial x_{i}}+k y_{j} \frac{\partial}{\partial y_{j}}\right) .
\end{aligned}
$$

Супераналоги полиномов Джека. Следуя Керову, Окунькову, Ольшанскому [13], определим супераналоги полиномов Джека. Рассмотрим алгебру многочленов $\mathcal{A}$ от бесконечного числа переменных $x_{1}, x_{2}, \ldots$ и $y_{1}, y_{2}, \ldots$ Пусть $p_{r}(x, y)=\sum x_{i}^{r}+\sum y_{j}^{r}$ - степенная сумма. Определим автоморфизм $\omega_{k}$ алгебры $\mathcal{A}$ по формуле

$$
\omega_{k}\left(p_{r}(x, y)\right)=\sum x_{i}^{r}-\frac{1}{k} \sum y_{j}^{r} .
$$

Пусть $P_{\lambda}(x, y, k)$ - обычный полином Джека. Тогда супераналог полинома Джека имеет вид

$$
S P_{\lambda}(x, y, k)=\omega_{k}\left(P_{\lambda}(x, y, k)\right) .
$$

Если положить $x_{n+1}=\cdots=y_{m+1}=\cdots=0$, то можно рассматривать супераналоги полиномов Джека от конечного числа переменных $S P_{\lambda}\left(x_{1}, \ldots, x_{n}, y_{1}, \ldots, y_{m}, k\right)$.

Теорема 1.1. Полиномы $S P_{\lambda}\left(x_{1}, \ldots, x_{n}, y_{1}, \ldots, y_{m}, k\right)$ являются собственными функциями оператора (1.11).

Сферические функции. В настоящей работе мы используем алгебраический подход к теории сферических функций. 
Пусть $\mathfrak{g}$ - конечномерная супералгебра Ли, $U(\mathfrak{g})$ - ее универсальная обертываюшая алгебра, $\mathfrak{b} \subset \mathfrak{g}$ - подалгебра. Пусть $\pi: \mathfrak{g} \rightarrow \mathfrak{g l}(V)$ - неприводимое представление, $V^{*}-$ дуальное представление, $v \in V$ - ненулевой $\mathfrak{b}$-инвариантньй вектор и $v^{*} \in V^{*}$ - также $\mathfrak{b}$-инвариантный вектор. Тогда матричный коэффициент $\theta^{\pi}\left(v^{*}, v\right) \in U(\mathfrak{g})^{*}$, где

$$
\theta^{\pi}\left(v^{*}, v\right)(u)=(-1)^{p(u) p(v)} v^{*}(\pi(u) v) \quad \forall u \in U(\mathfrak{g})
$$

будем называть сферической функиией, ассочиированной с тройкой $\left(\pi, v^{*}, v\right)$.

Пусть $L^{*}$ - левое корегулярное представление $\mathfrak{g}$; напомним, что оно задается формулой

$$
L^{*}(u) l(v)=(-1)^{p(u) p(l)} l\left({ }^{t} u v\right) \quad \forall u, v \in U(\mathfrak{g}),
$$

где $t$ - главный антиавтоморфизм $U(\mathfrak{g})$. Пусть $l \in U(\mathfrak{g})^{*}-$ двусторонне $\mathfrak{b}$-инвариантный функционал, т.е.

$$
l(x u)=l(u y)=0 \quad \forall x, y \in \mathfrak{b} \quad \text { и } u \in U(\mathfrak{g}) .
$$

Тогда $L^{*}(z) l$, где $z \in Z(\mathfrak{g})$, также является двусторонне $\mathfrak{b}$-инвариантным функционалом.

Пусть $\mathfrak{g}=\mathfrak{g l}(V) \oplus \mathfrak{g l}(V)$ и $\mathfrak{b} \simeq \mathfrak{g l}(V)$ - диагональная подалгебра, т.е. $\mathfrak{b}=\{(x, x) \mid$ $x \in \mathfrak{g l}(V)\}$. Пусть $\lambda$ - разбиение и $V^{\lambda}-$ неприводимый $\mathfrak{g l}(V)$-подмодуль в тензорной алгебре тождественного представления $\mathfrak{g l}(V)$, соответствуюший разбиению $\lambda$ (см. [14]). Тогда $\mathfrak{g}$-модуль $W^{\lambda}=V^{\lambda} \otimes\left(V^{\lambda}\right)^{*}$ неприводим и содержит единственный с точностью до постоянного множителя $\mathfrak{b}$-инвариантный вектор $v_{\lambda}$. Дуальньй модуль $\left(W^{\lambda}\right)^{*}$ содержит подобный вектор $v_{\lambda}^{*}$. Пусть $\varphi_{\lambda}=\theta^{\pi}\left(v_{\lambda}^{*}, v_{\lambda}\right)$ - соответствующая сферическая функция.

Пусть $\operatorname{dim} V=(n \mid m), I_{\overline{0}}=\{1, \ldots, n\}, I_{\overline{1}}=\{\overline{1}, \ldots, \bar{m}\},\left\{e_{i j} \mid i, j \in I=I_{\overline{0}} \coprod I_{\overline{1}}\right\}$ базис $\mathfrak{g l}(V)$, состоящий из матричных единиц, $\mathfrak{h}=\operatorname{Span}\left(e_{i i} \mid i \in I\right)$ - подалгебра Картана, состоящая из диагональных матрищ, и $\varepsilon_{1}, \ldots, \varepsilon_{n}, \varepsilon_{\overline{1}}, \ldots, \varepsilon_{\bar{m}}-$ левый дуальный базис к $\left\{e_{i i} \mid i \in I\right\}$ в $\mathfrak{h}^{*}$. Пусть $\mathfrak{h}^{+}=\{(x,-x) \mid x \in \mathfrak{h}\}$. Мы отождествляем $\mathfrak{h} \mathfrak{h}^{+}$, так же как и $\mathfrak{h}^{*} \mathrm{c}\left(\mathfrak{h}^{+}\right)^{*}$. Положим

$$
C_{2}=\sum_{i, j \in I}(-1)^{p(j)} e_{i j} e_{j i}
$$

Легко проверить, что $C_{2}$ - центральный элемент в обертывающей алгебре $\mathfrak{g l}(V)$ и в $\mathfrak{g}$, если $\mathfrak{g l}(V)$ рассматривается как первое слагаемое в $\mathfrak{g}$.

TеОРема 1.2. А. Каждий двусторонне инвариантный функиионал $l \in U(\mathfrak{g})^{*}$ однозначно определяется своим ограничением на $S\left(\mathfrak{h}^{+}\right)$.

Б. Пусть $\left(S\left(\mathfrak{h}^{+}\right)^{*}\right)^{\mathrm{inv}}$ - множество ограничений двусторонне инвариантных функционалов из $U(\mathfrak{g})^{*}$ на $S\left(\mathfrak{h}^{+}\right)$. Тогда для любого $z \in Z(\mathfrak{g})$ существует однозначно определенный оператор $\Omega_{z}$ на $\left(S\left(\mathfrak{h}^{+}\right)^{*}\right)^{\text {inv }}$. Он определяется по формуле

$$
\left(\Omega_{z} l^{\prime}\right)(u)=\left(L^{*}(z) l\right)(u)
$$

для любого $l^{\prime} \in\left(S\left(\mathfrak{h}^{+}\right)^{*}\right)^{\text {inv }}$ и его расширения $l \in U(\mathfrak{g})^{*}$. 
B. Вышеопределенный оператор $\Omega_{C_{2}}$, соответствующий $C_{2}$, совпадает с оператором $\mathcal{M}$, определенным в формуле (1.9), для $k=1$.

Г. Функиии $\varphi_{\lambda}$, как функциональ на $S\left(\mathfrak{h}^{+}\right)$, совпадают с точностью до постоянного множителя с полиномами $S P_{\lambda}(x, y ; 1)$, где $x_{i}=e^{\varepsilon_{i}}$ для $i \in I_{\overline{0}}$ u $y_{j}=e^{\varepsilon_{j}}$ для $j \in I_{\overline{1}}$.

Пусть $\mathfrak{g}=\mathfrak{g l}(V), \operatorname{dim} V=(n \mid m)$ и $m=2 r$ - четное число. Пусть $\mathfrak{b}=\mathfrak{o s p}(V)$-ортогонально-симплектическая подалгебра Ли в $\mathfrak{g l}(V)$, которая сохраняет тензор

$$
\sum_{i \in I_{\overline{0}}} e_{i}^{*} \otimes e_{i}^{*}+\sum_{j \in I_{\overline{1}}}\left(e_{\bar{j}}^{*} \otimes e \frac{*}{j+r}-e \frac{*}{j+r} \otimes e_{\bar{j}}^{*}\right) .
$$

Пусть $\psi$ - инволютивный автоморфизм $\mathfrak{g}$ такой, что он выделяет $\mathfrak{o s p}(V)$ :

$$
\mathfrak{o s p}(V)=\{x \in \mathfrak{g l}(V) \mid \psi(x)=-x\}
$$

Пусть $V^{\lambda}-\mathfrak{g}$-подмодуль в тензорной алгебре тождественного представления, отвечающий разбиению $\lambda$. Согласно [15] $V^{\lambda}$ содержит единственный с точностью до постоянного множителя $\mathfrak{b}$-инвариантный вектор $\tilde{v}_{\lambda}$ тогда и только тогда, когда $\lambda=2 \mu$, т.е. все строки $\lambda$ имеют четную длину. Вектор $\tilde{v}_{\lambda}^{*} \in\left(V^{\lambda}\right)^{*}$ определяется подобным образом. Пусть $\tilde{\varphi}_{\lambda}=\theta\left(v_{\lambda}^{*}, v_{\lambda}\right)$ - соответствующий матричный коэффициент. Положим $\mathfrak{h}^{+}=$ $\{x \in \mathfrak{h} \mid \psi(x)=x\}$, где $\mathfrak{h} \subset \mathfrak{g}$-подалгебра Картана.

Теорема 1.3. А. Кажсдый двусторонне инвариантный функционал на $U(\mathfrak{g})$ однозначно определяется своим ограничением на $S\left(\mathfrak{h}^{+}\right)$.

Б. Пусть $\left(S\left(\mathfrak{h}^{+}\right)^{*}\right)^{\text {inv }}$ - множество ограничений двусторонне инвариантных функиионалов. Тогда для каждого $z \in Z(\mathfrak{g})$ существует единственный оператор $\Omega_{z}$ на $\left(S\left(\mathfrak{h}^{+}\right)^{*}\right)^{\text {inv }}$. Он определяется по формуле

$$
\left(\Omega_{z} l^{\prime}\right)(u)=\left(L^{*}(z) l\right)(u)
$$

для любого $l^{\prime} \in\left(S\left(\mathfrak{h}^{+}\right)^{*}\right)^{\text {inv }}$ и его расширения $l \in U(\mathfrak{g})^{*}$.

B. Oператор $\Omega_{C_{2}}$, соответствующий $C_{2}$, совпадает с оператором $\mathcal{M}$, определеннылм в формуле (1.9), для $m=r$ и $k=1 / 2$.

Г. Функциональ $\tilde{\varphi}_{\lambda}$, как функции на $S\left(\mathfrak{h}^{+}\right)$, совпадают с точностью до постоянного множителя с полиномами $S P_{\mu}(x, y ; 1 / 2)$, әде $\lambda=2 \mu, \quad x_{i}=e^{2 \varepsilon_{i}}$ для $1 \leqslant i \leqslant n$ u $y_{j}=e^{2 \varepsilon_{j}} \partial \Omega_{\text {я }} 1 \leqslant j \leqslant r$.

Инвариантный интеграл. Для каждого $\mathfrak{g}$-модуля $W$ определим в $U(\mathfrak{g})^{*}$ подпространство $C(W)$, являющееся линейной оболочкой матричных коэффициентов $W$. Обозначим через $A(\mathfrak{g})$ подалгебру в $U(\mathfrak{g})^{*}$, порожденную матричными коэффициентами конечномерных представлений $W$, которые являются полупростыми при ограничении на $\mathfrak{g}_{\overline{0}}$. Пусть алгебра $\mathfrak{g}_{\overline{0}}$ редуктивна, ее представление на $\mathfrak{g}_{\overline{1}}$ полупросто, а представление на максимальной внешней степени $\mathfrak{g}_{\overline{1}}$ тривиально (равносильное требование: $\operatorname{tr}(\operatorname{ad} x)=0$ 
в модуле $\mathfrak{g}_{\overline{1}}$, если $\left.x \in \mathfrak{g}_{\overline{0}}\right)$. На $A(\mathfrak{g})$ определим скалярное произведение $\left\langle l_{1}, l_{2}\right\rangle=F\left(l_{1}^{t} l_{2}\right)$, где $l \mapsto l^{t}$ - главньй автоморфизм $U(\mathfrak{g})^{*}$ (он соответствует главному антиавтоморфизму $U(\mathfrak{g}))$. У уверждение А следуюшей теоремы - это основной результат работы [5]. Дополнительное утверждение, которое мы доказьваем, - это двусторонняя инвариантность интеграла.

Теорема 1.4. А. На $A(\mathfrak{g})$ существует и единственный с точностью до ненулевого постоянного множителя двусторонне инвариантный (относительно левого и правого корегулярных представлений) линейный функционал $F$ и $F(\varepsilon)=0$, где $\varepsilon$ - коединица в $U(\mathfrak{g})$.

Б. Если $V_{1}$ и $V_{2}$ - неизоморфные конечномерные простые $\mathfrak{g}$-модули, тогда $\left\langle l_{1}, l_{2}\right\rangle=0$ для любих $l_{1} \in C\left(V_{1}\right), l_{2} \in C\left(V_{2}\right)$.

B. Eсли $\operatorname{dim} V_{\overline{0}} \neq \operatorname{dim} V_{\overline{1}}, \operatorname{mozдa~}\left\langle l_{1}, l_{2}\right\rangle=0$ для любьх $l_{1}, l_{2} \in C(V)$.

\section{2. ДУАЛЬНОЕ СУПЕРПРОСТРАНСТВО К ОБЕРТЫВАЮШЕЙ АЛГЕБРЕ}

В этом разделе мы приводим некоторые факты, касающиеся супералгебр Ли, мы следуем при этом книге Диксмье [16]. Доказательства стандартны и поэтому опускаются. Пусть $\mathfrak{g}$ - супералгебра Ли, $U(\mathfrak{g})$ - ее обертывающая алгебра. Алгебра $U(\mathfrak{g})$ обладает каноническим антиавтоморфизмом $u \mapsto{ }^{t} u$, заданным на элементах супералгебры $\mathfrak{g}$ по правилу ${ }^{t} x=-x$. На элементы $U(\mathfrak{g})$ антиавтоморфизм можно распространить по правилу ${ }^{t}(u v)=(-1) p(u) p(v){ }^{t} v^{t} u$.

Мы наделяем $U(\mathfrak{g})^{*}$ структурой коалгебры:

$$
\begin{gathered}
c: U(\mathfrak{g}) \rightarrow U(\mathfrak{g}) \otimes U(\mathfrak{g}), \\
c(x)=x \otimes 1+1 \otimes x \quad \forall x \in \mathfrak{g} .
\end{gathered}
$$

Легко проверить, что ${ }^{t}(c(x))=c\left({ }^{t} x\right)$, где первый индекс $t$ - канонический антиавтоморфизм $U(\mathfrak{g}) \otimes U(\mathfrak{g}) \simeq U(\mathfrak{g} \oplus \mathfrak{g})$.

Лемма 2.1. Пусть $\operatorname{dim} \mathfrak{g}=(n \mid m)$. Тогда алгебра $U(\mathfrak{g})^{*}$ изоморфна алгебре формальных степенных рядов от $n$ четных и $m$ нечетных переменных.

Левое и правое корегулярные представления. Положим

$$
\begin{aligned}
& \left(L^{*}(u) l\right)(v)=(-1)^{p(u) p(l)} l\left({ }^{t} u v\right), \\
& \left(R^{*}(u) l\right)(v)=(-1)^{p(u)(p(l)+p(v))} l(v u) \quad \forall u, v \in U(\mathfrak{g}), \quad l \in U(\mathfrak{g})^{*} .
\end{aligned}
$$

Следующие утверждения легко проверяются:

1) $u \mapsto L^{*}(u)$ - представление $U(\mathfrak{g})$ в $U(\mathfrak{g})^{*}$ (называемое левым корегулярнылм представлением);

2) $u \mapsto R^{*}(u)$-представление $U(\mathfrak{g})$ в $U(\mathfrak{g})^{*}$ (называемое правы.м корегулярным представлением); 
3) $L^{*}(x)$ и $R^{*}(x)$ являются супердифференцированиями супералгебры $U(g)^{*}$.

Заметим также, что супералгебра $U(\mathfrak{g})^{*}$ обладает каноническим автоморфизмом $l \mapsto$ $l^{t}$, где $l^{t}(u)=l\left({ }^{t} u\right) \forall u \in U(\mathfrak{g}), l \in U(\mathfrak{g})^{*}$.

Матричные коэффициенты. Пусть $V-\mathfrak{g}$-модуль, $\pi: \mathfrak{g} \rightarrow \mathfrak{g l}(V)-$ соответствующее представление, $V^{*}$ - дуальный модуль. Для любых $v \in V$ и $v^{*} \in V^{*}$ определим линейную форму $\theta^{\pi}\left(v^{*}, v\right)$ на $U(\mathfrak{g})^{*}$, полагая

$$
\theta^{\pi}\left(v^{*}, v\right)(u)=(-1)^{p(u) p(v)} v^{*}(\pi(u) v) .
$$

Обозначим через $C(\pi)$ или $C(V)$ подпространство в $U(\mathfrak{g})^{*}$, порожденное $\theta^{\pi}\left(v^{*}, v\right)$ для всех $v \in V$ и $v^{*} \in V^{*}$.

ЛЕмма 2.2. A. $\theta^{\pi_{1} \otimes \pi_{2}}\left(v_{1}^{*} \otimes v_{2}^{*}, v_{1} \otimes v_{2}\right)=(-1)^{p\left(v_{1}\right) p\left(v_{2}^{*}\right)} \theta^{\pi_{1}}\left(v_{1}^{*}, v_{1}\right) \theta^{\pi_{2}}\left(v_{2}^{*}, v_{2}\right)$.

Б. $C\left(\pi_{1} \otimes \pi_{2}\right)=C\left(\pi_{1}\right) C\left(\pi_{2}\right)$.

В. Если $\pi$ - конечномерное представление, тогда

$$
\left(\theta^{\pi}\left(v^{*}, v\right)\right)^{t}=(-1)^{p(v) p\left(v^{*}\right)} \theta^{\pi^{*}}\left(v, v^{*}\right) .
$$

Будем рассматривать $U(\mathfrak{g})^{*}$ как $\mathfrak{g} \oplus \mathfrak{g}$-модуль относительно левого и правого корегулярных представлений. Тогда справедливы следующие леммы

Лемма 2.3. Отображсение $V^{*} \otimes V \rightarrow U(\mathfrak{g})^{*}$, заданное формулой $\left(v^{*}, v\right) \mapsto$ $\theta^{\pi}\left(v^{*}, v\right)$, является гомоморфизмом $\mathfrak{g} \oplus \mathfrak{g}-м о д у л е и ̆ . ~ Е с л и ~ \mathfrak{g - м о д у л ь ~} V$ неприводим, то вышеопределенное отображсене имеет тривиальное ядро.

Рассмотрим $\left(V^{*} \otimes V\right) \otimes\left(V^{*} \otimes V\right)$ как $\mathfrak{g} \oplus \mathfrak{g}$-модуль.

ЛЕмма 2.4. Пусть $V$ - неприводимый $\mathfrak{g}$-модуль. Тогда отображсение

$$
\begin{gathered}
\varphi: V^{*} \otimes V \otimes V^{*} \otimes V \rightarrow U\left(\mathfrak{g}^{*}\right), \\
\varphi\left(v_{1}^{*} \otimes v_{1} \otimes v_{2}^{*} \otimes v_{2}\right)=(-1)^{p\left(v_{1}^{*}\right) p\left(v_{2}^{*}\right)+p\left(v_{1}\right) p\left(v_{2}^{*}\right)+p\left(v_{1}^{*}\right) p\left(v_{1}\right)}\left(\theta^{\pi_{1}}\left(v_{2}^{*}, v_{1}\right)\right)^{t} \theta^{\pi_{2}}\left(v_{1}^{*}, v_{2}\right)
\end{gathered}
$$

является гомоморфизмом $\mathfrak{g} \oplus \mathfrak{g}$-модулей.

Лемма 2.5. Пусть $V$ - конечномерный $\mathfrak{g}$-модуль, $\pi$-соответствующее пред-



$$
\sum_{i}\left(\theta^{\pi}\left(v^{*}, v_{i}\right)\right)^{t} \theta^{\pi}\left(v_{i}^{*}, v\right)=\left(v^{*}, v\right) \varepsilon
$$



Пусть $\mathfrak{g}$ - супералгебра Ли такая, что алгебра $\mathfrak{g}_{\overline{0}}$ редуктивна, ее представление на $\mathfrak{g}_{\overline{1}}$ полупросто, а представление на максимальной внешней степени $\mathfrak{g}_{\overline{1}}$ тривиально. Пусть $A(\mathfrak{g})$ - подалгебра в $U(\mathfrak{g})^{*}$, порожденная матричными коэффициентами конечномерных представлений таких, что их ограничение на $\mathfrak{g}_{\overline{0}}$ полупросто. Легко проверить, что подалгебра $A(\mathfrak{g})$ инвариантна относительно левого и правого корегулярных представлений. В разделе 7 будет доказано сушествование на $A(\mathfrak{g})$ нетривиального и инвариантного относительно левого и правого корегулярных представлений функционала $F$ (интеграл Березина). 
Лемма 2.6. Пусть $W$ - неприводимое подпредставление $\pi: \mathfrak{g} \rightarrow T(V)$. Тогда:


для любих $w_{1}, w_{2} \in W$ и $w_{1}^{*}, w_{2}^{*} \in W^{*}$, где $d_{W}$ зависит только от $W$;

2) $n y с т ь \operatorname{dim} W_{\overline{0}} \neq \operatorname{dim} W_{\overline{1}}$, тогда $\left\langle l_{1}, l_{2}\right\rangle=0$ для любих $l_{1}, l_{2} \in C(W)$.

\section{3. СУПЕРАНАЛОГ ОПЕРАТОРА КАЛОДЖКРО}

Мы будем придерживаться здесь обозначений раздела 1 . Положим для $\alpha \in R$

$$
\begin{gathered}
\Delta_{\alpha}^{+}=e^{\frac{\alpha}{2}}+e^{-\frac{\alpha}{2}}, \quad \Delta_{\alpha}^{-}=e^{\frac{\alpha}{2}}-e^{-\frac{\alpha}{2}}, \\
\Delta_{1}=\prod_{\alpha \in R_{11}^{+}} \Delta_{\alpha}^{-}, \quad \Delta_{2}=\prod_{\beta \in R_{22}^{+}} \Delta_{\beta}^{-}, \quad \Delta_{12}=\prod_{\gamma \in R_{12}} \Delta_{\gamma}^{-}, \\
\delta^{(k)}=\Delta_{1}^{k} \Delta_{2}^{\frac{1}{k}} \Delta_{12}^{-1} .
\end{gathered}
$$

Определим оператор

$$
\mathcal{M}^{*}=\sum_{i=1}^{n} \partial_{i}^{2}-k \sum_{\bar{j}=1}^{m} \partial_{\bar{j}}^{2}+k \sum_{\alpha \in R_{11}^{+}} \frac{\Delta_{\alpha}^{+}}{\Delta_{\alpha}^{-}} \partial_{\alpha}-\sum_{\beta \in R_{22}^{+}} \frac{\Delta_{\beta}^{+}}{\Delta_{\beta}^{-}} \partial_{\beta}-\sum_{\gamma \in R_{12}} \frac{\Delta_{\gamma}^{+}}{\Delta_{\gamma}^{-}} \partial_{\gamma, k}
$$

где $\partial_{\alpha}, \partial_{\beta}, \partial_{\gamma, k}$ определены в (1.10).

ДоКАЗАТЕЛЬСТво ЛЕммы 1.1. Докажем равенство

$$
\delta^{(k)} \mathcal{M}^{*}\left(\delta^{(k)}\right)^{-1}=\mathcal{S} \mathcal{L}-\left(\rho_{k}, \rho_{k}\right)_{k},
$$

эквивалентное утверждению леммы 1.1. Следующие равенства легко проверяются:

$$
\begin{aligned}
\partial_{i}\left(\Delta_{\alpha}^{+}\right) & =\frac{1}{2} \alpha\left(e_{i}\right) \Delta_{\alpha}^{-}, \quad \partial_{i}\left(\Delta_{\alpha}^{-}\right)=\frac{1}{2} \alpha\left(e_{i}\right) \Delta_{\alpha}^{+}, \quad \alpha \in R, \\
\delta^{(k)} \partial_{i}\left(\delta^{(k)}\right)^{-1} & =\partial_{i}-\frac{k}{2} \sum_{\alpha \in R_{11}^{+}} \alpha\left(e_{i}\right) \frac{\Delta_{\alpha}^{+}}{\Delta_{\alpha}^{-}}+\frac{1}{2} \sum_{\gamma \in R_{12}} \gamma\left(e_{i}\right) \frac{\Delta_{\gamma}^{+}}{\Delta_{\gamma}^{-}}, \quad i \in I_{\overline{0}}, \\
\delta^{(k)} \partial_{i}\left(\delta^{(k)}\right)^{-1} & =\partial_{i}-\frac{1}{2 k} \sum_{\beta \in R_{22}^{+}} \beta\left(e_{i}\right) \frac{\Delta_{\beta}^{+}}{\Delta_{\beta}^{-}}+\frac{1}{2} \sum_{\gamma \in R_{12}} \gamma\left(e_{i}\right) \frac{\Delta_{\gamma}^{+}}{\Delta_{\gamma}^{-}}, \quad i \in I_{\overline{1}} .
\end{aligned}
$$

Оператор $\mathcal{M}^{*}$ может быть выражен в виде

$$
\begin{aligned}
\mathcal{M}^{*}= & \sum_{i \in I_{\overline{0}}} \partial_{i}^{2}-k \sum_{j \in I_{\overline{1}}} \partial_{j}^{2}+k \sum_{\alpha \in R_{11}^{+}, i \in I_{\overline{0}}} \alpha\left(e_{i}\right) \frac{\Delta_{\alpha}^{+}}{\Delta_{\alpha}^{-}} \partial_{i}- \\
& -\sum_{\beta \in R_{22}^{+}, j \in I_{\overline{1}}} \beta\left(e_{j}\right) \frac{\Delta_{\beta}^{+}}{\Delta_{\beta}^{-}} \partial_{j}-\sum_{\gamma \in R_{12}, i \in I_{\overline{0}}} \gamma\left(e_{i}\right) \frac{\Delta_{\gamma}^{+}}{\Delta_{\gamma}^{-}} \partial_{i}+ \\
& +k \sum_{\gamma \in R_{12}, j \in I_{\overline{1}}} \gamma\left(e_{j}\right) \frac{\Delta_{\gamma}^{+}}{\Delta_{\gamma}^{-}} \partial_{j} .
\end{aligned}
$$


Далее положим $X_{\alpha}=\Delta_{\alpha}^{+} / \Delta_{\alpha}^{-}$для $\alpha \in R^{+}$и пусть

$$
\begin{aligned}
\varphi_{i}=\sum_{\alpha \in R_{11}^{+}} \alpha\left(e_{i}\right) X_{\alpha}, & f_{i}=\sum_{\gamma \in R_{12}^{+}} \gamma\left(e_{i}\right) X_{\gamma}, \quad i \in I_{\overline{0}}, \\
h_{j} & =\sum_{\beta \in R_{22}^{+}} \beta\left(e_{j}\right) X_{\beta}, \quad g_{j}=\sum_{\gamma \in R_{12}^{+}} \gamma\left(e_{j}\right) X_{\gamma}, \quad j \in I_{\overline{1}} .
\end{aligned}
$$

Следуюшие равенства легко проверяются:

$$
\begin{aligned}
& \delta^{(k)} \partial_{i}^{2}\left(\delta^{(k)}\right)^{-1}=\partial_{i}^{2}+\left(f_{i}-k \varphi_{i}\right) \partial_{i}+\frac{1}{4}\left(f_{i}-k \varphi_{i}\right)^{2}+\frac{1}{2}\left(\partial_{i} f_{i}-k \partial_{i} \varphi_{i}\right), \quad i \in I_{\overline{0}}, \\
& \delta^{(k)} \partial_{j}^{2}\left(\delta^{(k)}\right)^{-1}=\partial_{j}^{2}+\left(g_{j}-\frac{1}{k} h_{j}\right) \partial_{j}+\frac{1}{4}\left(g_{j}-\frac{1}{k} h_{j}\right)^{2}+\frac{1}{2}\left(\partial_{j} g_{j}-\frac{1}{k} \partial_{j} h_{j}\right), \quad j \in I_{\overline{1}} .
\end{aligned}
$$

Следовательно, после несложных преобразований получим

$$
\begin{aligned}
\delta^{(k)} \mathcal{M}^{*}\left(\delta^{(k)}\right)^{-1}= & \sum_{i \in I_{\overline{0}}} \partial_{i}^{2}-2 k(k-1) \sum_{\alpha \in R_{11}} \frac{1}{\left(\Delta_{\alpha}^{-}\right)^{2}}-\left(k \rho_{1}, k \rho_{1}\right)_{1}- \\
& -k\left(\sum_{j \in I_{\overline{1}}} \partial_{j}^{2}-\frac{2}{k}\left(\frac{1}{k}-1\right) \sum_{\beta \in R_{22}} \frac{1}{\left(\Delta_{\beta}^{-}\right)^{2}}-\left(\frac{1}{k} \rho_{2}, \frac{1}{k} \rho_{2}\right)_{2}\right)+ \\
& +\left[-\frac{1}{4} \sum_{i \in I_{\overline{0}}} f_{i}^{2}+\frac{k}{2} \sum_{i \in I_{\overline{0}}} f_{i} \varphi_{i}+\frac{1}{2} \sum_{i \in I_{\overline{0}}} \partial_{i} f_{i}+\right. \\
& \left.+\frac{k}{4} \sum_{j \in I_{\overline{1}}} g_{j}^{2}-\frac{1}{2} \sum_{j \in I_{\overline{1}}} g_{j} h_{j}-\frac{k}{2} \sum_{j \in I_{\overline{1}}} \partial_{j} g_{j}\right] .
\end{aligned}
$$

Легко проверить, что $\partial_{i}\left(X_{\gamma}\right)=\gamma\left(e_{i}\right)\left(1-X_{\gamma}^{2}\right) / 2$. Остается преобразовать слагаемое в квадратных скобках (мы предполагаем, что $\gamma, \gamma_{1}, \gamma_{2} \in R_{12}, \alpha \in R_{11}^{+}, \beta \in R_{22}^{+}, i \in I_{\overline{0}}$, $j \in I_{\overline{1}}$, а также $\left\{\gamma_{1}, \gamma_{2}\right\}$ означает неупорядоченную пару различных элементов из $R_{12}$ ).

Легко проверить, что справедливо равенство

$$
\sum_{\left\{\gamma_{1}, \gamma_{2}\right\}}\left(\gamma_{1}, \gamma_{2}\right)_{2} X_{\gamma_{1}} X_{\gamma_{2}}+\sum_{\gamma, \alpha}(\gamma, \alpha)_{1} X_{\gamma} X_{\alpha}=\sum_{\left\{\gamma_{1}, \gamma_{2}\right\}}\left(\gamma_{1}, \gamma_{2}\right)_{2}+\sum_{\gamma, \alpha}(\gamma, \alpha)_{1}
$$

Аналогично доказьвается равенство

$$
\sum_{\left\{\gamma_{1}, \gamma_{2}\right\}}\left(\gamma_{1}, \gamma_{2}\right)_{1} X_{\gamma_{1}} X_{\gamma_{2}}+\sum_{\gamma, \beta}(\gamma, \beta)_{2} X_{\gamma} X_{\beta}=\sum_{\left\{\gamma_{1}, \gamma_{2}\right\}}\left(\gamma_{1}, \gamma_{2}\right)_{1}+\sum_{\gamma, \alpha}(\gamma, \beta)_{2}
$$


Следовательно,

$$
\begin{aligned}
& \delta^{(k) \mathcal{M}^{*}\left(\delta^{(k)}\right)^{-1}=} \sum_{i \in I_{\overline{0}}} \partial_{i}^{2}-2 k(k-1) \sum_{\alpha \in R_{11}} \frac{1}{\left(\Delta_{\alpha}^{-}\right)^{2}}-\left(k \rho_{1}, k \rho_{1}\right)_{1}- \\
&-k\left(\sum_{j \in I_{\overline{1}}} \partial_{j}^{2}-\frac{2}{k}\left(\frac{1}{k}-1\right) \sum_{\beta \in R_{22}} \frac{1}{\left(\Delta_{\beta}^{-}\right)^{2}}-\left(\frac{1}{k} \rho_{2}, \frac{1}{k} \rho_{2}\right)_{2}\right)- \\
&-2 \sum_{\left\{\gamma_{1}, \gamma_{2}\right\}} \frac{\left(\gamma_{1}, \gamma_{2}\right)_{k}}{\left(\Delta_{\gamma}^{-}\right)^{2}}+\frac{k}{2}\left(\sum_{\left\{\gamma_{1}, \gamma_{2}\right\}}\left(\gamma_{1}, \gamma_{2}\right)_{2}+\sum_{\gamma, \alpha}(\gamma, \alpha)_{1}\right)- \\
&-\frac{1}{2}\left(\sum_{\left\{\gamma_{1}, \gamma_{2}\right\}}\left(\gamma_{1}, \gamma_{2}\right)_{1}+\sum_{\gamma, \alpha}(\gamma, \beta)_{2}\right)-\frac{1}{4} \sum_{\gamma}(\gamma, \gamma)_{k}= \\
&= \sum_{i \in I_{\overline{0}}} \partial_{i}^{2}-2 k(k-1) \sum_{\alpha \in R_{11}} \frac{1}{\left(\Delta_{\alpha}^{-}\right)^{2}}-\left(k \rho_{1}, k \rho_{1}\right)_{1}- \\
&-k\left(\sum_{j \in I_{\overline{1}}} \partial_{j}^{2}-\frac{2}{k}\left(\frac{1}{k}-1\right) \sum_{\beta \in R_{22}} \frac{1}{\left(\Delta_{\beta}^{-}\right)^{2}}-\left(\frac{1}{k} \rho_{2}, \frac{1}{k} \rho_{2}\right)_{2}\right)_{-} \\
&-2 \sum_{\left\{\gamma_{1}, \gamma_{2}\right\}} \frac{\left(\gamma_{1}, \gamma_{2}\right)_{k}}{\left(\Delta_{\gamma}^{-}\right)^{2}}-\left(\rho_{12}, \rho_{12}\right)_{k}+\left(\rho_{12}, k \rho_{1}\right)_{k}-\left(\rho_{12}, \frac{1}{k} \rho_{2}\right)_{k}
\end{aligned}
$$

Лемма доказана.

\section{4. СУПЕРАНАЛОГИ ПОЛИНОМОВ ДЖЕКА}

Обычные полиномы Джека. Пусть $t_{1}, t_{2}, \ldots$ - последовательность независимых переменных, $\Lambda$ - алгебра симметрических функций, $\lambda$-разбиение, а $t^{\lambda} \equiv t_{1}^{\lambda_{1}} t_{2}^{\lambda_{2}} \ldots$. Мономиальные симметрические функции $m_{\lambda}$ являются суммой всех различных мономов, которые получаются из $t^{\lambda}$ перестановкой $t_{i}$. Можно определить степенные суммы $p_{r}=\sum_{i} t_{i}^{r}$ и $p_{\lambda}=p_{\lambda_{1}} p_{\lambda_{2}} \ldots$. Полиномы Джека $P_{\lambda}$ индексируются разбиениями и рационально зависят от параметра $k$ (мы используем параметр $k=1 / \alpha$, обратный параметру Макдональда). Они характеризуются следующими свойствами:

$$
\begin{gathered}
P_{\lambda}=m_{\lambda}+\text { члены меньшего лексикографического порядка, } \\
\left(P_{\lambda}, P_{\mu}\right)=0, \text { если } \lambda \neq \mu,
\end{gathered}
$$

где скалярное произведение определяется по формуле

$$
\left(p_{\lambda}, p_{\mu}\right)=\delta_{\lambda, \mu} k^{-l(\lambda)} z_{\lambda}, \quad z_{\lambda}=\prod i^{\mu_{i}}\left(\mu_{i} !\right)
$$

Полагая $t_{N+1}=t_{N+2}=\cdots=0$ в $P_{\lambda}$, мы можем рассматривать полиномы Джека от конечного числа переменных; они допускают другое описание. Пусть $t_{1}, \ldots, t_{N}$ - переменные и $u$ - дополнительная переменная. Рассмотрим семейство дифференциальных 
операторов $D(u, k)$, называемых операторами Секигучи и определяемых по формуле

$$
D(u, k)=\sum_{p=1}^{N} u^{p} D_{p}^{(k)}=V(t)^{-1} \operatorname{det}\left[t_{i}^{N-j}\left(t_{i} \partial t_{i}+(N-j) k+u\right)\right]_{1 \leqslant i \leqslant j \leqslant N}
$$

где $V(t)=\prod_{1 \leqslant i<j \leqslant N}\left(t_{i}-t_{j}\right)$. Тогда полиномы Джека $P_{\lambda}\left(t_{1}, \ldots, t_{N}\right)$, где $\lambda$-разбиение такое, что $\lambda_{N+1}=0$, однозначно определяются следуюшими свойствами:

1) $P_{\lambda}\left(t_{1}, \ldots, t_{N}\right)$ симметричен относительно $t_{1}, \ldots, t_{N}$;

2) $P_{\lambda}\left(t_{1}, \ldots, t_{N}\right)=t_{1}^{\lambda_{1}} \ldots t_{N}^{\lambda_{N}}+$ мономы меньшего лексикографического порядка;

3) $P_{\lambda}\left(t_{1}, \ldots, t_{N}\right)$ - собственная функшия операторов $D_{p}^{(k)}$. Более точно

$$
D(u, k) P_{\lambda}\left(t_{1}, \ldots, t_{N}\right)=\left(\prod_{i=1}^{N}\left(\lambda_{i}+(N-i) k+u\right)\right) P_{\lambda}\left(t_{1}, \ldots, t_{N}\right) .
$$

Положим

$$
\varphi(t)=\prod_{j=1}^{m}\left(1-y_{j} t\right) \prod_{i=1}^{n}\left(1-x_{i} t\right)^{-k}
$$

и $\varphi\left(t_{1}, \ldots, t_{N}\right)=\varphi\left(t_{1}\right) \ldots \varphi\left(t_{N}\right)=\varphi_{N}$.

Положим далее

$$
\begin{aligned}
\mathcal{H}= & \sum_{i=1}^{n}\left(x_{i} \frac{\partial}{\partial x_{i}}\right)^{2}-k \sum_{j=1}^{m}\left(y_{j} \frac{\partial}{\partial y_{j}}\right)^{2}+k \sum_{1 \leqslant i<j \leqslant n} \frac{x_{i}+x_{j}}{x_{i}-x_{j}}\left(x_{i} \frac{\partial}{\partial x_{i}}-x_{j} \frac{\partial}{\partial x_{j}}\right)- \\
& -\sum_{1 \leqslant i<j \leqslant m} \frac{y_{i}+y_{j}}{y_{i}-y_{j}}\left(y_{i} \frac{\partial}{\partial y_{i}}-y_{j} \frac{\partial}{\partial y_{j}}\right)- \\
& -\sum_{1 \leqslant i \leqslant n, 1 \leqslant j \leqslant m} \frac{x_{i}+y_{j}}{x_{i}-y_{j}}\left(x_{i} \frac{\partial}{\partial x_{i}}+k y_{j} \frac{\partial}{\partial y_{j}}\right) \\
\mathcal{L}_{N}= & \sum_{i=1}^{N}\left(t_{i} \frac{\partial}{\partial t_{i}}\right)^{2}+k \sum_{1 \leqslant i<j \leqslant N} \frac{t_{i}+t_{j}}{t_{i}-t_{j}}\left(t_{i} \frac{\partial}{\partial t_{i}}-t_{j} \frac{\partial}{\partial t_{j}}\right) .
\end{aligned}
$$

Следуюшая лемма подобна лемме 3.1 из работы [17].

ЛЕмма 4.1. Справедливо равенство

$$
\mathcal{H} \varphi_{N}-\mathcal{L}_{N} \varphi_{N}=(k(n-N)-m)\left(\sum_{i=1}^{n} t_{i} \frac{\partial}{\partial t_{i}}\right) \varphi_{N}
$$

ДОКАЗАТЕЛЬСТВО несложно провести индукшией по $N$. 
ДокАЗАТЕЛЬСТВО тЕОРЕМЫ 1.1. Легко проверить, что

$$
\omega_{k}\left(\frac{1}{\prod_{i, l}\left(1-x_{i} t_{l}\right)^{k} \prod_{j, l}\left(1-y_{j} t_{l}\right)^{k}}\right)=\frac{\prod_{j, l}\left(1-y_{j} t_{l}\right)}{\prod_{i, l}\left(1-x_{i} t_{l}\right)^{k}} .
$$

Тогда согласно тождеству Коши (см. [12])

$$
\frac{1}{\prod_{i, l}\left(1-x_{i} t_{l}\right)^{k} \prod_{j, l}\left(1-y_{j} t_{l}\right)^{k}}=\sum_{\lambda} \frac{1}{J_{\lambda}} P_{\lambda}(x, y, k) P_{\lambda}(t, k) .
$$

Применяя автоморфизм $\omega_{k}$, получим

$$
\omega_{k}\left(\frac{1}{\prod_{i, l}\left(1-x_{i} t_{l}\right)^{k} \prod_{j, l}\left(1-y_{j} t_{l}\right)^{k}}\right)=\sum_{\lambda} \frac{1}{J_{\lambda}} \omega_{k}\left(P_{\lambda}(x, y, k)\right) P_{\lambda}(t, k) .
$$

Следовательно,

$$
\frac{\prod_{j, l}\left(1-y_{j} t_{l}\right)}{\prod_{i, l}\left(1-x_{i} t_{l}\right)^{k}}=\sum_{\lambda} \frac{1}{J_{\lambda}} S P_{\lambda}(x, y, k) P_{\lambda}(t, k)
$$

и

$$
\varphi_{N}=\sum_{\lambda_{N+1}=0} \frac{1}{j_{\lambda}} S P_{\lambda}\left(x_{1}, \ldots, x_{n}, y_{1}, \ldots, y_{m}, k\right) P_{\lambda}\left(t_{1}, \ldots, t_{N}, k\right) .
$$

Теперь согласно лемме 4.1 выполняется равенство (4.4). Введем оператор

$$
\mathcal{L}_{N}^{*}=\mathcal{L}_{N}+(k(n-N)-m)+\left(\sum_{i=1}^{n} t_{i} \frac{\partial}{\partial t_{i}}\right)
$$

Тогда

$$
\begin{aligned}
\sum_{\lambda_{N+1}=0} & \frac{1}{j_{\lambda}} \mathcal{H}\left(S P_{\lambda}\left(x_{1}, \ldots, x_{n}, y_{1}, \ldots, y_{m}, k\right)\right) P_{\lambda}\left(t_{1}, \ldots, t_{N}, k\right)= \\
\quad= & \sum_{\lambda_{N+1}=0} \frac{1}{j_{\lambda}} S P_{\lambda}\left(x_{1}, \ldots, x_{n}, y_{1}, \ldots, y_{m}, k\right) \mathcal{L}^{*}\left(P_{\lambda}\left(t_{1}, \ldots, t_{N}, k\right)\right) .
\end{aligned}
$$

Известно, что $P_{\lambda}\left(t_{1}, \ldots, t_{N}, k\right)$ являются собственными функциями оператора $\mathcal{L}^{*}$. Следовательно, $S P_{\lambda}\left(x_{1}, \ldots, x_{n}, y_{1}, \ldots, y_{m}, k\right)$ являются собственными функциями оператора $\mathcal{H}$. Теорема доказана. 


\section{5. СФЕРИЧЕСКИЕ ФУНКЦИИ И РАДИАЛЬНЫЕ ЧАСТИ ОПЕРАТОРОВ ЛАПЛАСА ДЛЯ ПАРЫ $(\mathfrak{g l} \oplus \mathfrak{g l} \mathfrak{g l})$}

Пусть $\mathfrak{g}=\mathfrak{g l}(V)$ - супералгебра Ли линейных преобразований $(n \mid m)$-мерного суперпространства $V, \mathfrak{h}$ - подалгебра Картана, $R$ - система корней, $U(\mathfrak{g})$ - обертьваюшая алгебра и $U(\mathfrak{g})^{*}$ - дуальное пространство, наделенное структурой супералгебры. Для любого ad-инвариантного функционала на пространстве $U(\mathfrak{g})$ (т.е. для любого $l$ такого, что $\left.l(u, v)=(-1)^{p(u) p(v)} l(v, u)\right)$ обозначим через $\varphi_{l}$ производящую функцию его ограничения на $S(\mathfrak{h})$, именно

$$
\varphi_{l}\left(t_{1}, \ldots, t_{n}\right)=\sum \frac{l\left(e_{11}^{\nu_{1}} \ldots e_{n n}^{\nu_{n}}\right)}{\left(\nu_{1}\right) ! \ldots\left(\nu_{n}\right) !} t_{1}^{\nu_{1}} \ldots t_{n}^{\nu_{n}}
$$

На $S(\mathfrak{h})^{*}$ определим следуюшие операторы для любого $f \in S(\mathfrak{h})$ :

$$
\left(\partial_{i} l\right)(f)=l\left(e_{i i} f\right), \quad\left(D_{i j} l\right)(f)=l\left(e_{i j} e_{j i} f\right) .
$$

Лемма 5.1. Пусть $\alpha=\varepsilon_{i}-\varepsilon_{j}$. Тогда

$$
D_{i j}=\frac{e^{\alpha}}{e^{\alpha}-1}\left(\partial_{i}-(-1)^{p(i)+p(j)} \partial_{j}\right)
$$

ДокАЗАТЕЛЬСтвО. Действительно,

$$
\begin{aligned}
\left(D_{i j} l\right)(f) & =l\left(f e_{i j} e_{j i}\right)=l\left(e_{i j} f(h+\alpha(h)) e_{j i}\right)=(-1)^{p(i)+p(j)} l\left(f(h+\alpha(h)) e_{j i} e_{i j}\right)= \\
& =l\left(f(h+\alpha(h)) e_{i j} e_{j i}\right)-l\left(f(h+\alpha(h))\left[e_{i j}, e_{j i}\right]\right)= \\
& =\left(e^{\alpha} D_{i j} l\right)(f)-l\left(f(h+\alpha(h))\left(e_{i i}-(-1)^{p(i)+p(j)} e_{j j}\right)\right)= \\
& =\left(e^{\alpha} D_{i j}-e^{\alpha}\left(\partial_{i}-(-1)^{p(i)+p(j)} \partial_{j}\right)\right)(l)(f) .
\end{aligned}
$$

Лемма доказана.

Лемма 5.2. Пусть $\mathfrak{g}-$ супералгебра Ли, $\mathfrak{b}=\{(x, x) \mid x \in \mathfrak{g}\}-$ ее диагональная подалгебра, $I$ - левый идеал в $U(\mathfrak{g} \oplus \mathfrak{g})$, порожденный $\mathfrak{b}$ и $M=U(\mathfrak{g} \oplus \mathfrak{g}) / I$. Пусть $\sigma: \mathfrak{g} \rightarrow \mathfrak{g} \oplus \mathfrak{g}-$ вложение в первое слагаемое, т.е. $\sigma(x)=(x, 0)$. Пусть $\tilde{\sigma}: U(\mathfrak{g}) \rightarrow M$ - отображение, индуцированное гомоморфизмом $U(\mathfrak{g}) \rightarrow U(\mathfrak{g} \oplus \mathfrak{g})$, которое продолжает $\sigma$, и $\rho(x)=(x, x)-$ изоморфизм $\mathfrak{g} c \mathfrak{b}$. Тогда $\tilde{\sigma}([x, u])=\rho(x) \tilde{\sigma}(u)$.

СлЕДСтвИЕ 5.1. Алгебра функиионалов на $U(\mathfrak{g} \oplus \mathfrak{g})$, двусторонне инвариантных относительно $\mathfrak{b}$, изоморфна алгебре функиионалов на $U(\mathfrak{g})$, инвариантных относительно присоединенного действия.

ДокАЗАТЕЛЬСТво сводится к прямой проверке. 
ЛЕмма 5.3. Пусть $\mathfrak{g}=\mathfrak{g l}(V), \mathfrak{h}-$ подалгебра Картана в $\mathfrak{g}$, тогда

$$
U(\mathfrak{g})=S(\mathfrak{h})+[U(\mathfrak{g}), U(\mathfrak{g})]
$$

ДокАЗАТЕЛЬСтво. Любой элемент из $U(\mathfrak{g})$ может быть представлен как сумма элементов вида $f X_{\alpha_{1}} \ldots X_{\alpha_{r}}$, где $f \in S(\mathfrak{h}), \alpha_{i} \in R(R-$ система корней $\mathfrak{g}), X_{\alpha_{i}}$ - элемент веса $\alpha_{i}$. Следовательно, для доказательства леммы достаточно показать, что для $r>0$ $f X_{\alpha_{1}} \ldots X_{\alpha_{r}} \in[U(g), U(g)]$. Проведем индукцию по $r$.

Если $r=1$, тогда $\left[X_{\alpha}, f\right]=(f(h-\alpha(h))-f(h)) X_{\alpha}=R_{\alpha}(f) X_{\alpha}$. Однако, как легко видеть, любой элемент из $S(\mathfrak{h})$ может быть представлен как $R_{\alpha}(f)$. Если $r>1$, тогда

$$
\begin{aligned}
& {\left[X_{\alpha_{1}}, f X_{\alpha_{2}} \ldots X_{\alpha_{r}}\right]=\left[X_{\alpha_{1}}, f\right] X_{\alpha_{2}} \ldots X_{\alpha_{r}}+f\left[X_{\alpha_{1}}, X_{\alpha_{2}}\right] X_{\alpha_{3}} \ldots X_{\alpha_{r}}+} \\
& +(-1)^{p\left(X_{\alpha_{1}}\right) p\left(X_{\alpha_{2}}\right)} f X_{\alpha_{2}}\left[X_{\alpha_{1}}, X_{\alpha_{3}}\right] X_{\alpha_{4}} \ldots X_{\alpha_{r}}+ \\
& +(-1)^{p\left(X_{\alpha_{1}}\right)\left(p\left(X_{\alpha_{2}}\right)+p\left(X_{\alpha_{3}}\right)\right)} f X_{\alpha_{2}} X_{\alpha_{3}}\left[X_{\alpha_{1}}, X_{\alpha_{4}}\right] X_{\alpha_{5}} \ldots X_{\alpha_{r}}+\cdots \text {. }
\end{aligned}
$$

Но $\left[X_{\alpha}, f\right] X_{\alpha}=R_{\alpha}(f) X_{\alpha}$, и по предположению индукции $f X_{\alpha_{1}} \ldots X_{\alpha_{r}} \in[U(\mathfrak{g}), U(\mathfrak{g})]$. Лемма доказана.

ДокаЗАТЕЛЬСтво теоремы 1.2. Лемма 5.3 сразу влечет утверждение А. Утверждение Б очевидно. Докажем утверждение В. Пусть $l$ - инвариантный функционал на $U(\mathfrak{g}), \varphi_{l}$ - производящая функция его ограничения на $S(\mathfrak{h})$. Тогда с учетом леммы 5.2 получим

$$
\begin{aligned}
\Omega\left(\varphi_{l}\right)= & \left(\sum_{i, j \in I}(-1)^{p(j)} e_{i j} e_{j i}\right) \varphi_{l}=\left(\sum_{i \in I_{\overline{0}}} \partial_{i}^{2}-\sum_{j \in I_{\overline{1}}} \partial_{j}^{2}\right) \varphi_{l}+ \\
& +\sum_{\alpha \in R^{+}}\left((-1)^{p(j)} \frac{e^{\alpha}}{e^{\alpha}-1}\left(\partial_{i}-(-1)^{p(i)+p(j)} \partial_{j}\right)+\right. \\
& \left.+(-1)^{p(i)} \frac{e^{-\alpha}}{e^{-\alpha}-1}\left(\partial_{j}-(-1)^{p(i)+p(j)} \partial_{i}\right)\right) \varphi_{l}= \\
= & \left(\sum_{i \in I_{\overline{0}}} \partial_{i}^{2}-\sum_{j \in I_{\overline{1}}} \partial_{j}^{2}-\sum_{\alpha \in R^{+}} \frac{1+e^{\alpha}}{1-e^{\alpha}}\left((-1)^{p(j)} \partial_{i}-(-1)^{p(i)} \partial_{j}\right)\right) \varphi_{l}
\end{aligned}
$$

Докажем утверждение Г. Как легко проверить, $\theta=\sum_{i \in I} e_{i} \otimes e_{i}^{*}-\mathfrak{b}$-инвариант, при условии, что $\left\{e_{i}\right\}_{i \in I}-$ базис в $V$ и $\left\{e_{i}^{*}\right\}_{i \in I}-$ его левый дуальный базис. Аналогично $\theta^{*}=\sum_{i \in I} e_{i}^{*} \otimes e_{i}-$ также $\mathfrak{b}$-инвариант.

$\mathrm{C}$ точностью до постоянного множителя

$$
\varphi_{\lambda}(u)=\theta^{\pi}\left(\left(\theta^{*}\right)^{\otimes p}, \theta^{\otimes p}\right)(u)=\left(\theta^{*}\right)^{\otimes p}\left(\left(e_{\lambda} \times e_{\lambda}\right) u \theta^{\otimes p}\right) \quad \forall u \in U(g),
$$

где $e_{\lambda}$ - идемпотент в симметрической группе, соответствуюший разбиению $\lambda$, и $\varphi_{\lambda}(u)$ пропорционально $\left(\theta^{*}\right)^{\otimes p}\left(e_{\lambda} \times 1 u \theta^{\otimes p}\right)$. 
Разлагая

$$
e_{\lambda}=\frac{1}{p !} \sum \chi^{\lambda}(\sigma) \sigma
$$

где $\chi^{\lambda}$ - характер соответствуюшего представления $S_{p}$, мы видим, что благодаря тождеству

$$
\theta^{\pi_{1} \otimes \pi_{2}}\left(v_{1}^{*} \otimes v_{2}^{*}, v_{1} \otimes v_{2}\right)=(-1)^{p\left(v_{1}\right) p\left(v_{2}^{*}\right)} \theta^{\pi_{1}}\left(v_{1}^{*}, v_{1}\right) \theta^{\pi_{2}}\left(v_{2}^{*}, v_{2}\right)
$$

достаточно рассмотреть случай, когда $\sigma$ - цикл $(1,2, \ldots, p)$. Пусть $\left\{e_{i i}\right\}_{i \in I}-$ базис $\mathfrak{h}$ и $\left\{\varepsilon_{i}\right\}_{i \in I}$ - левый дуальный базис; пусть $e^{l}$ обозначает гомоморфизм $S(\mathfrak{h})$ в поле комплексных чисел, продолжающий линейную форму $l$. Достаточно учитывать только слагаемые, у которых $i_{1}=i_{2}=\cdots=i_{p}$, в соотношении

$$
\theta^{\otimes p}=\sum_{i_{1}, \ldots, i_{p} \in I} e_{i_{1}} \otimes e_{i_{1}}^{*} \otimes \cdots \otimes e_{i_{p}} \otimes e_{i_{p}}^{*}
$$

Следовательно, если $\sigma=(1,2, \ldots, p)$, тогда

$$
\begin{aligned}
\left(\theta^{*}\right)^{\otimes p}\left(u \sigma \theta^{\otimes p}\right) & =\left(\theta^{*}\right)^{\otimes p}\left(\sum_{i \in I}\left(e_{i} \otimes e_{i}^{*}\right)^{\otimes p}\right)=\sum_{i \in I}\left(\theta^{*}\right)^{\otimes p}\left(u \sigma \theta^{\otimes p}\right)= \\
& =\sum_{i \in I}\left(\theta^{*}\right)^{\otimes p}\left((-1)^{(p-1) i} u\left(e_{i} \otimes e_{i}^{*}\right)^{\otimes p}\right)= \\
& =\sum_{i \in I}(-1)^{(p-1) i} e^{p \varepsilon_{i}}(u)\left(\theta^{*}\right)^{\otimes p}\left(e_{i} \otimes e_{i}^{*}\right)= \\
& =\sum_{i \in I}(-1)^{i} e^{p \varepsilon_{i}}(u)=\sum_{i \in I_{\overline{0}}} x_{i}^{p}-\sum_{j \in I_{\overline{1}}} y_{j}^{p}
\end{aligned}
$$

Это означает, что с точностью до постоянного множителя $\varphi_{\lambda}=\sum\left(\chi_{\mu}^{\lambda} / Z_{\mu}\right) s p_{\mu}$, где $\chi_{\mu}^{\lambda}$ - значение характера симметрической группы на элементе циклового типа $\mu, Z_{\mu}=$ $\prod i^{\mu_{i}}\left(\mu_{i}\right) !$ и

$$
s p_{\mu}=\left(\sum_{i \in I_{\overline{0}}} x_{i}^{\mu_{1}}-\sum_{j \in I_{\overline{1}}} y_{j}^{\mu_{1}}\right)\left(\sum_{i \in I_{\overline{0}}} x_{i}^{\mu_{2}}-\sum_{j \in I_{\overline{1}}} y_{j}^{\mu_{2}}\right) \ldots
$$

Поэтому $\varphi_{\lambda}$ с точностью до постоянного множителя совпадает с $S P_{\lambda}(x, y, 1)$. Теорема доказана.

\section{6. СФЕРИЧЕСКИЕ ФУНКЦИИ И РАДИАЛЬНЫЕ ЧАСТИ ОПЕРАТОРОВ ЛАПЛАСА ДЛЯ ПАРЫ $(\mathfrak{g l}, \mathfrak{o s p})$}

Пусть $\mathfrak{g}=\mathfrak{g l}(V)$, где $\operatorname{dim} V=(n \mid 2 r)$. Пусть также $I_{\overline{0}}=\{1, \ldots, n\}$ и $I_{\overline{1}}=\{\overline{1}, \ldots, \overline{2 r}\}$, a $\left\{e_{i j}\right\}_{i, j \in I}$ - базис, состоящий из матричных единиц в $\mathfrak{g l}(V)$. Легко проверить, что антиавтоморфизм супертранспонирования в $\mathfrak{g l}(V)$ имеет вид

$$
{ }^{t} e_{i j}=(-1)^{p(i)(p(j)+1)} e_{j i}
$$


Далее положим $\varepsilon(i)=1$, если $i \in I_{\overline{0}} \cup\{\overline{r+1}, \ldots, \overline{2 r}\}$, и $\varepsilon(i)=-1$, если $i \in\{\overline{1}, \ldots, \bar{r}\}$. Положим $\delta(i)=i+\bar{r}(\bmod \overline{2 r})$, если $i \in I_{\overline{1}}$, и $\delta(i)=i$, если $i \in I_{\overline{0}}$. Теперь определим оператор $S$ :

$$
S e_{i}=\varepsilon(i) e_{\delta(i)} .
$$

Ясно, что $S^{2}=J$, где $J$ - оператор четности в $V$, т.е. $J e_{i}=(-1)^{p(i)} e_{i}$ и, следовательно, $S J=J S$, более того, ${ }^{t} S=S J=J S=S^{3}=S^{-1}$. Для любого $x \in \mathfrak{g l}(V)$ положим $\psi(x)=S^{t} x S^{-1}$. Несложно проверить, что $\psi$ - инволютивный антиавтоморфизм ассоциативной супералгебры $\operatorname{Mat}(V)$, т.е. $\psi^{2}=1$ и $\psi(x y)=(-1)^{p(x) p(y)} \psi(y) \psi(x)$, причем $\psi\left(e_{i j}\right)=(-1)^{p(i) p(j)} \varepsilon(j) \varepsilon(\delta(i)) e_{\delta(j) \delta(i)}$. Легко видеть, что $\mathfrak{g}=\mathfrak{g}^{-} \oplus \mathfrak{g}^{+}$, где $\mathfrak{g}^{-}=\{x \in$ $\mathfrak{g} \mid \psi(x)=-x\}$ и $\mathfrak{g}^{+}=\{x \in g \mid \psi(x)=x\}$. Заметим, что $\mathfrak{g}^{-}$- подалгебра Ли в $\mathfrak{g l}(V)$, изоморфная $\mathfrak{o s p}(V)$, и $\mathfrak{g}^{+}-\mathfrak{g}^{-}$-модуль. Для любого $x \in \mathfrak{g}$ положим

$$
x^{+}=\frac{1}{2}(x+\psi(x)), \quad x^{-}=\frac{1}{2}(x-\psi(x)),
$$

что соответствует разложению $\mathfrak{g}=\mathfrak{g}^{-} \oplus \mathfrak{g}^{+}$.

Пусть $\mathfrak{h}$ - подалгебра Картана в $\mathfrak{g}$ и $\mathfrak{h}^{+}=\operatorname{Span}\left(e_{i i}^{+} \mid i \in I\right)$.

Лемма 6.1. Для $f \in S\left(\mathfrak{h}^{+}\right)$и $\alpha=\varepsilon_{i}-\varepsilon_{j}$ положим

$$
R_{i j}^{-} f=\frac{1}{2}[f(h-\alpha(h))-f(h+\alpha(h))], \quad R_{i j}^{+} f=\frac{1}{2}[f(h-\alpha(h))+f(h+\alpha(h))] .
$$

Тогда справедливы следующие равенства:

1) $e_{i j}^{-} f=R_{i j}^{+} f e_{i j}^{-}+R_{i j}^{-} f e_{i j}^{+}$

2) $R_{i j}^{-} f e_{i j} e_{j i}-\left(R_{i j}^{-}-R_{i j}^{+}\right) f \cdot\left[e_{i j}^{-}, e_{j i}^{+}\right] \in \mathfrak{g}^{-} U(\mathfrak{g})+U(\mathfrak{g}) \mathfrak{g}^{-}$;

3) $\left[e_{i j}^{-}, e_{i j}^{+}\right]=\left(e_{i i}^{+}-(-1)^{p(i)+p(j)} e_{j j}^{+}\right) / 2$;

4) если $h \in \mathfrak{h} u \alpha=\varepsilon_{i}-\varepsilon_{j}$, тогда

$$
\left[h, e_{i j}^{+}\right]=\alpha(h) e_{i j}^{-}, \quad\left[h, e_{i j}^{-}\right]=\alpha(h) e_{i j}^{+} .
$$

ДОКАЗАТЕЛЬСТво сводится к прямой проверке.

Лемма 6.2. Пусть $I=\mathfrak{g}^{-} U(\mathfrak{g})+U(\mathfrak{g}) \mathfrak{g}^{-}$. Тогда $U(\mathfrak{g})=S\left(\mathfrak{h}^{+}\right)+I$.

ДокАЗАТЕЛЬСтво. Достаточно показать, что для $q>0 u=f e_{\alpha_{1}}^{+} \ldots e_{\alpha_{q}}^{+} \in I$, где $f \in S\left(\mathfrak{h}^{+}\right)$и $e_{\alpha_{1}}^{+}, \ldots, e_{\alpha_{q}}^{+} \in \mathfrak{g}^{+}$являются весовыми векторами. Проведем индукцию по $q$. Если $q=1$ и $f \in S\left(\mathfrak{h}^{+}\right)$, то согласно лемме 6.1, п. 1 имеем $R_{i j}^{-} f e_{i j}^{+}=e_{i j}^{-} f-R_{i j}^{+} f e_{i j}^{-}$. Следовательно, $R_{i j}^{-} f e_{i j}^{+} \in I$, поэтому $f e_{i j}^{+} \in I$. Пусть $q>1$. Тогда

$$
\begin{aligned}
R_{\alpha_{1}}^{-} f e_{\alpha_{1}}^{+} \ldots e_{\alpha_{q}}^{+} & =e_{\alpha_{1}}^{-} f e_{\alpha_{2}}^{+} \ldots e_{\alpha_{q}}^{+}-R_{\alpha_{1}}^{+} f e_{\alpha_{1}}^{-} e_{\alpha_{2}}^{+} \ldots e_{\alpha_{q}}^{+} \equiv \\
& \equiv-R_{\alpha_{1}}^{+} f e_{\alpha_{1}}^{-} e_{\alpha_{2}}^{+} \ldots e_{\alpha_{q}}^{+}(\bmod I) \equiv R_{\alpha_{1}}^{+} f \cdot\left[e_{\alpha_{1}}^{-}, e_{\alpha_{2}}^{+} \ldots e_{\alpha_{q}}^{+}\right]= \\
& =-R_{\alpha_{1}}^{+} f \cdot\left[e_{\alpha_{1}}^{-}, e_{\alpha_{2}}^{+}\right] e_{\alpha_{3}}^{+} \ldots e_{\alpha_{q}}^{+}-R_{\alpha_{1}}^{+} f e_{\alpha_{2}}^{+}\left[e_{\alpha_{1}}^{-}, e_{\alpha_{3}}^{+}\right] \ldots e_{\alpha_{q}}^{+}+\cdots \in I .
\end{aligned}
$$

Лемма доказана. 
Лемма 6.3. Пусть $\alpha=\varepsilon_{i}-\varepsilon_{j}, j \neq \delta(i), l$-двусторонне $\mathfrak{b}$-инвариантный функиионал на $U(\mathfrak{g})$ и $\varphi_{l}$ - производящая функция его ограничения на $S\left(\mathfrak{h}^{+}\right)$. Пусть $D_{i j}(l)(f)=l\left(f e_{i j} e_{j i}\right), \partial_{i}^{+}(l)(f)=l\left(f e_{i i}^{+}\right)$, где $f \in S\left(\mathfrak{h}^{+}\right)$. Тогда

$$
D_{i j}=\frac{e^{\alpha}}{e^{\alpha}-e^{-\alpha}}\left(\partial_{i}^{+}-(-1)^{p(i)+p(j)} \partial_{j}^{+}\right) .
$$

ДокАЗАТЕЛЬСТво является следствием утверждения 2 леммы 6.1.

ДокаЗАТЕЛЬство теоремы 1.3. Утверждение А следует из леммы 6.2. Докажем утверждение Б. Пусть $\sum_{i, j \in I}(-1)^{p(j)} e_{i j} e_{j i}-$ оператор Лапласа для $\mathfrak{g l}(V)$. Согласно лемме 6.3 радиальная часть его ограничения на $S\left(\mathfrak{h}^{+}\right)$имеет вид (мы исключаем корни $\beta=\varepsilon_{i}-\varepsilon_{\delta(i)}$, так как $\left.e_{i \delta(i)}^{+}=0\right)$

$$
\begin{aligned}
\mathcal{M}= & \sum_{i=1}^{n} \partial_{i}^{2}-\sum_{\bar{j}=1}^{2 r} \partial_{j}^{2}+\sum_{\alpha \in R_{11}^{+}} \frac{e^{\alpha}+e^{-\alpha}}{e^{\alpha}-e^{-\alpha}} \partial_{\alpha}^{+}- \\
& -\sum_{\beta \in R_{22}^{+}} \frac{e^{\beta}+e^{-\beta}}{e^{\beta}-e^{-\beta}} \partial_{\beta}^{+}-\sum_{\gamma \in R_{12}} \frac{e^{\gamma}+e^{-\gamma}}{e^{\gamma}-e^{-\gamma}} \partial_{\gamma, 1}^{+},
\end{aligned}
$$

где $\partial_{\alpha}=\partial_{i}^{+}-\partial_{j}^{+}, \partial_{\beta}=\partial_{\bar{i}}^{+}-\partial_{\bar{j}}^{+}, \partial_{\gamma, 1}=\partial_{\bar{i}}^{+}-\partial_{\bar{j}}^{+}$.

Заметим теперь, что $\left.\varepsilon_{i}\right|_{\mathfrak{h}^{+}}=\left.\varepsilon_{\delta(i)}\right|_{\mathfrak{h}}+$. Поэтому выражение $(6.3)$ принимает вид

$$
\begin{aligned}
\mathcal{M}= & \sum_{i=1}^{n} \partial_{i}^{2}-\sum_{j=1}^{r} \partial_{j}^{2}+\sum_{\alpha \in R_{11}^{+}} \frac{e^{2 \alpha}+1}{e^{2 \alpha}-1} \partial_{\alpha}^{+}- \\
& -4 \sum_{\beta \in \frac{1}{4} R_{22}^{+}} \frac{e^{2 \beta}+1}{e^{2 \beta}-1} \partial_{\beta}^{+}-2 \sum_{\gamma \in \frac{1}{2} R_{12}} \frac{e^{2 \gamma}+1}{e^{2 \gamma}-1} \partial_{\gamma, 1}^{+},
\end{aligned}
$$

где

$\frac{1}{4} R_{22}=\left\{\varepsilon_{i}-\varepsilon_{j} \mid i, j \in \frac{1}{2} I_{\overline{1}}\right\}, \quad \frac{1}{2} R_{12}=\left\{\varepsilon_{i}-\varepsilon_{j} \mid i \in I_{\overline{0}}, j \in \frac{1}{2} I_{\overline{1}}\right\}, \quad \frac{1}{2} I_{\overline{1}}=\{\overline{1}, \ldots, \bar{r}\}$.

Положим $T\left(e^{l}\right)=e^{l+l_{\overline{0}}}$, где $l_{\overline{0}}-$ четная часть $l$. Тогда, как легко проверить,

$$
T^{-1} \partial_{i}^{+} T=\left\{\begin{array}{l}
\partial_{i}^{+}, \quad \text { если } p(i)=\overline{1}, \\
2 \partial_{i}^{+}, \quad \text { если } p(i)=\overline{0},
\end{array} \quad T^{-1} e^{l} T=e^{l_{\overline{1}}+\frac{1}{2} l_{\overline{0}}} .\right.
$$

Следовательно, эти преобразования приводят формулу (6.4) к виду

$$
\begin{aligned}
\mathcal{M}= & 4 \sum_{i=1}^{n} \partial_{i}^{2}-2 \sum_{j=1}^{r} \partial_{\bar{j}}^{2}+\sum_{\alpha \in R_{11}^{+}} \frac{e^{\alpha}+1}{e^{\alpha}-1} 2 \partial_{\alpha}^{+}- \\
& -4 \sum_{\beta \in \frac{1}{4} R_{22}^{+}} \frac{e^{\beta}+1}{e^{\beta}-1} \partial_{\beta}^{+}-4 \sum_{\gamma \in \frac{1}{2} R_{12}} \frac{e^{2 \gamma}+1}{e^{2 \gamma}-1} \partial_{\gamma, \frac{1}{2}}^{+}
\end{aligned}
$$


Последнее выражение равно $4 \mathcal{M}_{2}$.

Теперь докажем утверждение В. Вначале опишем инвариантный вектор $v_{\lambda} \in V_{\lambda}$, где все строки диаграммы $\lambda$ имеют четную длину. Легко проверить, что

$$
\theta=\sum_{i \in I} \varepsilon(i) e_{i} \otimes e_{\delta(i)} \quad \text { и } \theta^{*}=\sum_{i \in I} \varepsilon(i) e_{i}^{*} \otimes e_{\delta(i)}^{*}
$$

являются $\mathfrak{b}$-инвариантами. Следовательно, $\theta^{\otimes p}$ - также $\mathfrak{b}$-инвариант. С точностью до постоянного множителя

$$
\varphi_{\lambda}(u)=\theta^{\pi}\left(\left(\theta^{*}\right)^{\otimes p}, \theta^{\otimes p}\right)(u)=\left(\theta^{*}\right)^{\otimes p}\left(e_{\lambda} u \theta^{\otimes p}\right) \quad \forall u \in U(\mathfrak{g}),
$$

где $e_{\lambda}-$ примитивный идемпотент в алгебре Гекке $\left(S_{2 p}, H_{p}\right)$. Явная форма $e_{\lambda}$ известна [11]:

$$
e_{\lambda}=\sum \frac{\omega_{\mu}^{\lambda}}{Z_{2 \mu}} \sigma
$$

где $\sigma$ пробегает множество представителей двойных классов смежности.

Если $\mu=\left(\mu_{1}, \ldots, \mu_{q}\right)$, мы можем предполагать, что $\sigma$ имеет цикловую структуру $\left(2 \mu_{1}, \ldots, 2 \mu_{q}\right)$. Теперь вычислим функционал $\varphi_{\lambda, \mu}(u)=\left(\theta^{*}\right)^{\otimes p}\left(\sigma u \theta^{\otimes p}\right)$, где $u \in S(\mathfrak{h})$. Согласно тождеству (5.3) достаточно предполагать, что $\sigma$ - цикл четной длины $2 p$. Имеem

$$
\theta^{\otimes p}=\sum \varepsilon(\psi) e_{\psi}, \quad\left(\theta^{*}\right)^{\otimes p}=\sum \varepsilon(\psi) e_{\psi}^{*},
$$

где сумма берется по всем отображениям

$$
\psi:[1, \ldots, 2 p] \longrightarrow[1, \ldots, n, \overline{1}, \ldots, \overline{2} r] \text { и } \delta(\psi(2 i))=\psi(2 i-1) \quad \text { для } i=1, \ldots, l
$$

и

$$
e_{\psi}=e_{\psi(1)} \otimes \cdots \otimes e_{\psi(2 p)}, \quad \varepsilon(\psi)=\varepsilon(\psi(1)) \varepsilon(\psi(3)) \ldots \varepsilon(\psi(2 l-1)) .
$$

Если $\sigma$ - цикл, то

$$
\left(\theta^{*}\right)^{\otimes p}\left(u \sigma \theta^{\otimes p}\right)=\left(\sum_{i \in I_{\overline{0}}}\left(e^{2 \varepsilon_{i}}\right)^{p}-2 \sum_{j \in \frac{1}{2} I_{\overline{1}}}\left(e^{2 \varepsilon_{j}}\right)^{p}\right)(u) .
$$

Поэтому, полагая $x_{i}=e^{2 \varepsilon_{i}}, i \in I_{\overline{0}}, y_{j}=e^{2 \varepsilon_{j}}, j \in J_{\overline{1}} / 2$, получим

$$
\varphi_{\lambda}=\sum \frac{\omega_{\mu}^{\lambda}}{Z_{2 \mu}} s p_{\mu}\left(x, y, \frac{1}{2}\right)
$$

где

$$
s p_{\mu}\left(x, y, \frac{1}{2}\right)=s p_{\mu_{1}}\left(x, y, \frac{1}{2}\right) \ldots s p_{\mu_{q}}\left(x, y, \frac{1}{2}\right), \quad s p_{l}=\sum_{i \in I_{\overline{0}}} x_{i}^{l}-2 \sum_{j \in \frac{1}{2} I_{\overline{1}}} y_{j}^{l} .
$$

Теорема доказана. 


\section{7. АЛГЕБРАИЧЕСКИЙ АНАЛОГ ИНТЕГРАЛА БЕРЕЗИНА}

Для обычных полиномов Джека, соответствуюших алгебре Ли $\mathfrak{g l}(n)$, существует скалярное произведение, индуцированное инвариантным интегралом на $U(n)$. Березин построил [18] инвариантный интеграл на унитарной супергруппе $U(n \mid m)$ и установил ряд его свойств. В частности, матричные коэффициенты любого конечномерного неприводимого представления $V$ такого, что $\operatorname{dim} V_{\overline{0}} \neq \operatorname{dim} V_{\overline{1}}$, являются изотропными относительно скалярного произведения, возникающего из интеграла Березина. В работе Шенерта и Занга [5] доказано сушествование и единственность инвариантного интеграла для супералгебр Ли $\mathfrak{g}$ с условием, что алгебра $\mathfrak{g}_{\overline{0}}$ редуктивна, ее представление на $\mathfrak{g}_{\overline{1}}$ полупросто, а представление на максимальной внешней степени $\mathfrak{g}_{\overline{1}}$ тривиально. Доказательство Шенерта и Занга основано на теории алгебр Хопфа. В этом разделе передоказан этот результат без использования теории алгебр Хопфа и доказана двусторонняя инвариантность интеграла из работы [5].

Пусть $\mathfrak{g}$ - конечномерная супералгебра Ли, причем алгебра $\mathfrak{g}_{\overline{0}}$ редуктивна, а ее представление на $\mathfrak{g}_{\overline{1}}$ полупросто. Обозначим через $A(\mathfrak{g})$ линейное подпространство в $U(\mathfrak{g})^{*}$, равное сумме $C(V)$, где $V$ - конечномерные представления $\mathfrak{g}$, ограничение которых на $\mathfrak{g}_{\overline{0}}$ полупросто. Поскольку

$$
C\left(V_{1}\right) \otimes C\left(V_{2}\right)=C\left(V_{1}\right) C\left(V_{2}\right), \quad C\left(V_{1} \oplus V_{2}\right)=C\left(V_{1}\right)+C\left(V_{2}\right),
$$

то $A(\mathfrak{g})$ - подалгебра в $U(\mathfrak{g})^{*}$. Легко проверить, что $A(\mathfrak{g})$ инвариантна относительно левого и правого корегулярных представлений и является полупростым $\mathfrak{g}_{\overline{0}}$-модулем относительно каждого из них. Пусть $V_{0}-$ неприводимый конечномерный $\mathfrak{g}_{0}$-модуль. Обозначим через $A(\mathfrak{g})_{V_{0}}^{n}$ изотипическую компоненту типа $V_{0}$ относительно правого корегулярного представления в $A(\mathfrak{g})$.

Лемма 7.1. Пусть $V=\operatorname{Ind}_{\mathfrak{g}_{\overline{0}}}^{\mathfrak{g}}\left(V_{0}\right)$. Рассмотрим каноническое отображение $\theta$

$$
V^{*} \otimes V \rightarrow U(g)^{*}, \quad\left(v^{*}, v\right) \mapsto \theta\left(v^{*}, v\right) .
$$

Тогда ограничение $\theta$ на $V^{*} \otimes V_{0}$ является изоморфизмом $V^{*} \otimes V_{0} u A(\mathfrak{g})_{V_{0}}^{n}$.

ДоКАЗАТЕЛЬСТво. Согласно лемме 2.3 отображение $\theta$ является гомоморфизмом $\mathfrak{g} \oplus \mathfrak{g}$-модулей. Поэтому $\theta\left(V^{*} \otimes V_{0}\right) \subset A(\mathfrak{g})_{V_{0}}^{n}$. Докажем обратное включение. Пусть $W_{0}$ - подмодуль относительно правого корегулярного представления в $U(\mathfrak{g})^{*}$, изоморфный $V_{0}$. Тогда имеем гомоморфизм $\mathfrak{g}_{0}$-модулей $\varphi_{0}: V_{0} \rightarrow U(\mathfrak{g})^{*}$ и его продолжение $\varphi: V \rightarrow$ $U(\mathfrak{g})^{*}$. Определим $v_{\varphi}^{*} \in V^{*}$ по формуле $v_{\varphi}^{*}(v)=\varphi(v)(1)$. Легко проверить, что $\theta\left(v_{\varphi}^{*}, v\right)=$ $\varphi(v)$. Поэтому $\theta\left(V^{*} \otimes V_{0}\right)=A(\mathfrak{g})_{V_{0}}^{n}$. Осталось показать, что ограничение $\theta$ на $V^{*} \otimes V_{0}$ имеет тривиальное ядро. Заметим, что отображение $\theta$ является двойственным к отображению $\pi: U(\mathfrak{g}) \rightarrow \operatorname{End}(V)$, которое определяет структуру $\mathfrak{g}$-модуля на $V$, если мы отождествляем $\operatorname{End}(V)$ с $V \otimes V^{*}$, a $\operatorname{End}(V)^{*}$ с $V^{*} \otimes V$. Поэтому инъективность ограничения $\theta$ равносильна тому, что образ $\pi$ содержит $\operatorname{Hom}\left(V_{0}, V\right)$. Из неприводимости $V_{0}$ как $\mathfrak{g}_{\overline{0}}$-модуля следует сюръективность отображения $U\left(\mathfrak{g}_{\overline{0}}\right) \rightarrow \operatorname{Hom}\left(V_{0}\right)$. Отсюда следует, что образ $\pi$ содержит $\operatorname{Hom}\left(V_{0}, V\right)$. Лемма доказана. 
СЛЕДСТВИЕ 7.1. Пусть $\mathfrak{g}$ - конечномерная супералгебра Ли, алгебра $\mathfrak{g}_{\overline{0}}$ редуктивна, ее представление на $\mathfrak{g}_{1}$ полупросто, а представление на максимальной внешней степени $\mathfrak{g}_{1}$ тривиально. Тогда на алгебре $A(\mathfrak{g})$ существует нетривиальный, единственный с точностью до постоянного множсителя функционал, инва риантный относительно левого корегулярного представления.

ДокАЗАТЕЛЬСТво. Согласно лемме 7.1

$$
A(\mathfrak{g})=\bigoplus\left(\operatorname{Ind}_{\mathfrak{g}_{\overline{0}}}^{\mathfrak{g}} V_{0}\right)^{*} \otimes V_{0}
$$

где $V_{0}$ принадлежит множеству представителей всех неприводимых конечномерных представлений $\mathfrak{g}_{\overline{0}}$. Пусть $\pi_{0}$ - тривиальное представление $\mathfrak{g}_{\overline{0}}$ и $p$ - проекция $A(\mathfrak{g})$ на $A(\mathfrak{g})_{\pi_{0}}^{n}$ параллельно подпространству $K=\bigoplus\left(\operatorname{Ind}_{\mathfrak{g}_{0}}^{\mathfrak{g}} V_{0}\right)^{*} \otimes V_{0}$, где $V_{0} \neq \pi_{0}$. Далее, согласно лемме $7.1 A(\mathfrak{g})_{\pi_{0}}^{n}$ как $\mathfrak{g} \oplus \mathfrak{g}_{\overline{0}}$-модуль изоморфин $\left(\operatorname{Ind}_{\mathfrak{g}_{0}}^{\mathfrak{g}}\left(\pi_{0}\right)\right)^{*} \otimes \pi_{0}$. Поэтому двойственное пространство к $A(\mathfrak{g})_{\pi_{0}}^{n}$ как $\mathfrak{g} \oplus \mathfrak{g}_{\overline{0}}$-модуль изоморфно $\operatorname{Ind}_{\mathfrak{g}_{0}}^{\mathfrak{g}}\left(\pi_{0}\right) \otimes \pi_{0}$, и согласно лемме 5.2 из работы [15] $\left(\operatorname{Ind}_{\mathfrak{g}_{0}}^{\mathfrak{g}} V_{0}\right)^{\mathfrak{g}}$ как векторное пространство изоморфно $V_{0}^{\mathfrak{g}_{\overline{0}}}$. Следовательно, на $A(\mathfrak{g})_{\pi_{0}}^{n}$ существует единственный с точностью до константы нетривиальный линейньй функционал $l$, инвариантный относительно $\mathfrak{g} \oplus \mathfrak{g}_{\overline{0}}$. Поэтому функционал $F=l \circ p$ является $\mathfrak{g} \oplus \mathfrak{g}_{\overline{0}}$-инвариантным функционалом на $A(\mathfrak{g})$. Таким образом, доказано сушествование левоинвариантного функционала. Докажем единственность. Пусть $F_{1}$ - левоинвариантный функционал на $A(\mathfrak{g})$. Тогда $F_{1} \circ \theta$ является инвариантным функционалом на $\left(\operatorname{Ind}_{\mathfrak{g}_{\overline{0}}}^{\mathfrak{g}}\left(V_{0}\right)\right)^{*} \otimes V_{0}$ (второй сомножитель мы рассматриваем как тривиальный $\mathfrak{g}$-модуль). Следовательно, $F_{1} \circ \theta$ определяет $\mathfrak{g}$-инвариантный элемент модуля $\operatorname{Ind}_{\mathfrak{g}_{\overline{0}}}^{\mathfrak{g}}\left(V_{0}\right)$ и, следовательно, $\mathfrak{g}_{\overline{0}}$-инвариантный элемент модуля $V_{0}$. Поэтому $F_{1}\left(A(\mathfrak{g})_{V_{0}}^{n}\right)=0$, если $V_{0} \neq \pi_{0}$. Следовательно, $F_{1}=\alpha F$, где $\alpha$ - комплексное число. Докажем теперь, что функционал $F$ правоинвариантен. Пусть $u \in U(\mathfrak{g})$, тогда функционал $F_{1}(a)=F\left(R^{*}(u) a\right)$ также левоинвариантен. Следовательно, $F \circ R^{*}(u)=\alpha(u) F$, где $\alpha$ - одномерное представление $U(\mathfrak{g})$. Но как было показано, функционал $F$ инвариантен относительно $\mathfrak{g}_{\overline{0}}$ справа, поэтому $\alpha$ - тривиальное представление, и, следовательно, $F$ двусторонне инвариантен. Следствие доказано.

ДокАЗАТЕЛЬСТво тЕоРЕмЫ 1.4. Утверждение А об инвариантном функционале сразу вытекает из следствия 7.1. Пусть $\varepsilon-$ коединица, тогда $\varepsilon \in A(\mathfrak{g})_{\pi_{0}}^{n}$ и не является образующей этого модуля. Следовательно, $F(\varepsilon)=0$.

Утверждения Б и В сразу следуют из леммы 2.4 .

Благодарности. Автор благодарен Г. Ольшанскому за совет рассматривать однородные суперпространства при построении супераналога оператора Калоджеро и Д. Лейтесу за помощь, а также International Newton Institute (Кембридж) за финансовую поддержку. 


\section{Список литературы}

[1] A. Sergeev. J. Nonlinear Math. Phys. 2001. V. 8. P. 59.

[2] L. Brink, T. Hanson, S. Konstein, M. Vasiliev. Nucl. Phys. B. 1993. V. 401. P. 591.

[3] P. Desrosiers, L. Lapointe, P. Mathieu. Supersymmetric Calogero-Moser-Sutherland model and Jack superpolynomials. hep-th/0103178.

[4] P. Desrosiers, L. Lapointe, P. Mathieu. Jack superpolynomials, superpartition ordering and determinantal formulas. hep-th/0105107.

[5] M. Scheunert, R. Zhang. Integration on Lie supergroups. math.RA/0012052.

[6] В. Серганова. Функц. анализ и его прилож. 1983. Т. 17. № 3. С. 46.

[7] M. Olshanetsky, A. Perelomov. Phys. Rep. 1983. V. 94. № 6. P. 313.

[8] А. П. Веселов, М. В. Фейгин, О. А. Чальцх. УМН. 1996. Т. 51. № 3. С. 185.

[9] O. Chalykh, M. Feigin, A. Veselov. Commun. Math. Phys. 1999. V. 206. № 3. P. 533.

[10] L. Lapointe, L. Vinet. Commun. Math. Phys. 1996. V. 178. № 2. P. 425.

[11] I. Macdonald. Symmetric functions and Hall polynomials. Oxford: Clarendon, 1995.

[12] R. Stanley. Adv. Math. 1996. V. 77. P. 76.

[13] S. Kerov, A. Okounkov, G. Olshanski. Int. Math. Res. Notices. 1998. № 4. P. 173.

[14] A. Сергеев. Матем. сб. 1984. Т. 165. С. 422.

[15] A. Sergeev. Michigan J. Math. 2001. V. 49. P. 113.

[16] ЖК. Диксмье. Универсальные обертывающие алгебры. М.: Мир, 1978.

[17] S. Sahi. Int. Math. Res. Notices. 1996. V. 20. P. 997.

[18] $\Phi$. А. Березин. Введение в алгебру и анализ с антикоммутирующими переменными. М.: Изд-во МГУ, 1983. 